\title{
Transcendental Logic, One and Itself, First-Order Logic and Number: The Problem of Metaphysics in the Critique of Pure Reason
}

\author{
Kazuhiko Yamamoto* \\ Megumi Institute of Ethics and Philosophy, Japan
}

*Corresponding Author: Kazuhiko Yamamoto, Megumi Institute of Ethics and Philosophy, Japan

\begin{abstract}
Our metaphysical exploration - an attempt to rectify the foundation of Kant's "transcendental philosophy" - led us to the findings that 1) number, which belongs to the category of allness, signifies the unity of the categories, 2) number is nothing but the product of the transcendental synthesis of the imagination in conjunction with the synthesis of apprehension and the synthesis of representations - synthesis speciosa and synthesis intellectualis - 3) the transcendental synthesis of the imagination, which stands under synthesis speciosa and synthesis intellectualis could operate in virtue of the regressive synthesis itself, yielding possibility itself of the category of community - number " 1 " or prime number or one and itself. Therefore, number, i.e., pure schema of magnitude is subject to the law of everything alterable in its spacetime-determination that everything that happens must find its cause in the appearances (of the previous state), which is space-time itself.
\end{abstract}

Keywords: One thing, Number, Relation-Numbers, First-Order Logic, Causality

\section{INTRODUCTION}

The metaphysical exploration - an attempt to rectify the foundation of Kant's "transcendental philosophy" $\left(\mathrm{B} 29^{1}\right)$ - led us to the findings that while "appearances in general are nothing outside our representations" (A507), appearances themselves are things that exist outside our representations (YAMAMOTO 2018: 20-45). So far, we have already clarified that "appearances themselves (e.g., humans) must be subsumed under the category, but only under the schemata of disappearance of 'the things themselves, which appear' (B324). Since the objects to which these principles are to be related are things in themselves, it is possible to cognize anything about them synthetically a priori. Now, it is nothing but appearances themselves whose complete cognition, to which in the end all a priori principles must come down, is the 'possibility of experience' (A156) or 'possible experience' (A489/B517). Consequently these principles can have as their goal the conditions of the unity of empirical cognition in the synthesis of the appearances. However, since these conditions of the unity are to be thought only in the schema of nullity in space-time, the category contains the function, unrestricted by any sensible condition, of their unity, as of a synthesis in general. We think that 'the function, unrestricted by any sensible condition, of their unity, as of a synthesis in general' (B224) pertains to space-time itself" (YAMAMOTO 2018: 20-45). Therefore, we have said that "1) the schema of nullity in space-time - 'the objects of nature itself' (A114) - is equivalent to 'the original causes of things' (A318), i.e., space-time itself, 2) the categories - the 'object of all possible experience' (A114) or the conditions of the unity, which are 'thought only in the schema of the pure concept of the understanding' (B224) - are the causality, 3) space-time itself - the category - and filled space-elapsing time - the categories - signify 'principles' - the cause and the causality" (YAMAMOTO 2018: 20-45). This insight would open the way to whole new realms of mathematics and philosophy, and is expected to enable us to find a solution to the conundrums in mathematics and physics.

${ }^{1}$ B29 designates the pagination of the standard German edition of Kant's works, as indicated by means of marginal numbers in the Critique of Pure Reason (Kant, Immanuel, Critique of Pure Reason, Cambridge University Press, 1999). All citations are the same. 
When we try to find a solution to conundrums in metaphysics and science, this process must go on along with the exploration in which we attempt to find an answer to the question: where, when, and why a seed of the conundrums was originally embedded in metaphysics and science. Furthermore, this exploration involves an effort to clarify how philosophers or scientists have tried to find a solution to the conundrums through metaphysics or logic or mathematics. We have already clarified that one of the causes of conundrums in mathematics resides in the fact that "when geometry and mathematics, as a science, have appeared among humans, inserting points or lines or numbers into nullity in space-time (EUCLID 2002: 1-36), a number - quantum continuum - has been alienated from 'the appearance as quantum continuum' (A527/B555)" (YAMAMOTO 2017d: 19-29). Kant makes a discourse on how numbers emerged among humans in primordial times, saying, "as I take the fingers of my hand as an intuition for assistance with the concept of 5, to that image of mine I now add the units that I have previously taken together in order to constitute the number 5 one after another to the number 7, and thus see the number 12 arise" (B15-B16). We have to stress that there is a decisive difference between the Kant's way of thinking and ours in such a way that, in our way of thinking, "one (homogeneous) unit - the number ' 1 ' - is correspondent to 'the pure image of all magnitudes (quantorum), ' i.e., either a pure image of a human in filled space-elapsing time - quantum discretum - or a pure image of a deceased in nullity in space-time - quantum continuum" (YAMAMOTO 2018: 20-45), while in Kant's way of thinking, number " 1 " is meant to be a finger - a filled space-elapsing time - which does not comprise a pure image of a finger in nullity in space-time. From our way of thinking in regard to number "1," it follows that "1) since the product of "the successive addition of one (homogeneous) unit to another' is equivalent to nullity in space-time - 'one complete whole' (A676/B704), i.e., quantum continuum - the product of the successive addition of 'one complete whole' to space-time itself is the same as the number ' 1,2 ' 2 ) since number ' 1 ' is commensurate with 'a representation that summarizes the successive addition of one (homogeneous) unit to another' (B182), number '2' is also a representation that summarizes the successive addition of one (homogeneous) unit to another, and so on. We can repeat it infinitely since the product of 'the successive addition of one (homogeneous) unit to another' or the successive addition of 'one complete whole' to another is equivalent to nullity in space-time, i.e., space-time itself - the number ' 1 .' 'Thus number is nothing other than the unity of the synthesis of the manifold of a homogeneous intuition in general' (B182-A143)" (YAMAMOTO 2018: 20-45). In contrast to numbers, which arise through our way of thinking, numbers which arise through the Kant's way of thinking would cause a serious problem, about which Frege explicitly states that, "If we try to produce the number by putting together different distinct objects, the result is an agglomeration in which the objects contained remain still in possession of precisely those properties which serve to distinguish them from one another; and that is not the number" (FREGE 1980: 39-67). When Kant came across the facts that 1) "if we try to produce the number by putting together different distinct objects, the result is an agglomeration..." (FREGE 1980: 39-67), 2) insofar as it is believed that "a figure is that which is contained by any boundary or boundaries" (EUCLID 2002: 1-36), putting together different distinct figures results in an "agglomeration," 3) this would intractably lead him to "the antinomy of pure reason in its cosmological ideas" (A506/B534), he could do nothing but create his "things in themselves" or noumena in an attempt to efface "the antinomy of pure reason" (YAMAMOTO 2016: 87-100, YAMAMOTO 2017a: 19-37). Ironically, the Kant's attempt to rescue the universe from the abyss of antinomy seems to have driven mathematics into further difficulty. In regard to this issue, we have made a discourse, saying, "the 'pure schema of magnitude (quantitatis)' or '(homogeneous) unit' number - signifies a figure among nullity in space-time, whose image is conjured up in humans' brain in virtue of 'mathematical-transcendental ideas' (A529/B557). Since 'appearances themselves' (A104) - empty space-nullified time or filled space-elapsing time - is commensurate with 'the transcendental ideality of appearances' (A506/B534), they would last forever irrespective of the existence of humans on this planet" (YAMAMOTO 2017d: 19-29). "Because Kant annuls 'mathematical-transcendental ideas' (A529/B557) by means of the 'transcendental dialectic' (B282), it follows that 'a likeness of things which appear in the world of sense' or 'the multiplicity of units' cannot be assumed as quantum discretum any more. We think that insofar as the 'mathematicaltranscendental ideas' rest on Kant's 'thing in itself' (A676/B704), 'natural or real numbers' in mathematics cannot have the properties of categories. 'Numerical formulas' (B206) cease to signify the 'transcendental principle of the mathematics of appearances' (B206)" (YAMAMOTO 2017d: 1929). When Kant was "forced to take refuge in intuition, as indeed geometry always does" (B65-A48), 
he had deprived numbers of "mathematical-transcendental ideas" by means of creating "things in themselves," annulling the "transcendental principle of the mathematics" of appearances themselves in "numerical formulas."

Apparently, Kant, who came across the "antinomy of pure reason," thinks that "a special act of the understanding is requisite" (B111), which enables humans to think that "a number," which belongs to "the category of allness" (B111), is equivalent to "the unity of the categories" (A249). If "a special act of the understanding" is meant to be Kant's thing in itself, we feel concern for "a number." Furthermore, we have concern about Kant's way of thinking, which says, "I take the fingers of my hand as an intuition for assistance with the concept of 5" (B15). We think that there are two reasons for the Kant's way of thinking that "a special act of the understanding is requisite." One reason is the issue of ethics, about which we have already discussed (YAMAMOTO 2016: 87-100, YAMAMOTO 2017a: 19-37). The second reason is that while Kant's discourse in regard to the "mathematical categories" (B110) or the "dynamical ones" (B110) in the "Table of Categories" (B106) shows us a way through which we can attain "synthetic a priori cognition" (A14/B28), it incessantly leads Kant to the discourse that the "pure category does not suffice for any synthetic a priori principle" (A248). In regard to the "Table of Categories" (B106), "when Kant says, "insofar as it rests on a priori concepts, and dividing it mathematically in accordance with determinate principles,...' (B109), we have to say that the first class belongs among the dynamical categories or metaphysical categories, while the second are the mathematical ones" (YAMAMOTO 2017d: 19-29). Since the dynamical categories or metaphysical categories, which are to signify space-time itself (YAMAMOTO 2017b: 72-81, YAMAMOTO 2017d: 19-29, YAMAMOTO 2018: 20-45), are nothing but "the a priori concepts of space and time" (B57) "in accordance with which unity of experience is to arise from perceptions" (A180) or "anticipation of perception" (B217) as "a perception itself" (A180). They are, as "a rule" and "a principle," to "be valid of the objects (of the appearances) constitutively" (A180) and regulatively. Since "mathematical categories" are to stand under the dynamical categories or metaphysical categories, we dare to say that mathematical categories - "number," e.g., "a priori categories" (B159) and "numerical formulas" (B206), i.e., a pure category - suffice for "synthetic $a$ priori principle." While the whole discourse in the Critique of Pure Reason is directed to the elucidation of a "constitutive principle of reason" (A509/B537) - a "constitutive principle of appearances in themselves" (A516/B544) - and the "regulative principle of reason" (A517/B545) by means of the "mathematical categories," Kant seems not to have achieved his aim (YAMAMOTO 2018: 20-45). We think that the issue of "mathematical categories" and the "dynamical categories" in the "Table of Categories" is crucial in order to find a solution to conundrums in mathematics and physics. Therefore this issue needs to be discussed more.

\section{Geometry, Synthetic Proposition, And Synthetic a Priori Proposition}

When Kant says that "the propositions of geometry are cognized synthetically a priori and with apodictic certainty" (B64-A47), he seems to have believed that "the self-evident propositions" (B205) in geometry, which states that "with three lines, two of which taken together are greater than the third, a triangle can be drawn" (B205), correspond to "synthetic a priori propositions" (B205) - "axioms" (B205). This is a very strange discourse. Even if it is proclaimed that "the propositions of geometry are cognized synthetically a priori and with apodictic certainty," and even if they appear to be "absolutely necessary and universally valid truths" (A47), they cannot be grounded on the "synthetic a priori cognitions of things" (A247). Why can they not be? It is because there has been no geometry itself in the world of sense. Therefore, "geometry" must "take refuge in intuition" (B65) in order for it to hold the status of synthetic a priori propositions. Geometry is requested to pertain to "an object in intuition" (A48). Kant explains it, saying, "You must therefore give your object a priori in intuition, and ground your synthetic proposition on this" (A48). What does to "give your object a priori in intuition" mean? It means that "the rule of its synthesis at the same time yields this intuition a priori in every empirical example" (B221), which is tantamount to saying that "rules of a mathematical synthesis" (B221) and "numerical magnitudes" (B221) should pertain to "realitas noumenon" (B320) - "a priori intuition" (B40) or nullity in space-time. If "all propositions of geometry" (A47) are to pertain to "a priori intuition," the "synthetic proposition" in regard to geometry can be thought to signify "pure a priori intuition" (A48). Under this assumption, it is merely proclaimed that "all geometrical cognition is immediately evident because it is grounded on intuition a priori, and the 
objects are given through the cognition itself a priori in intuition (as far as their form is concerned)" (B120-A88). Here, we feel serious concern. Where does a human encounter "the triangle in itself" (A48) in the world of sense? Since there is no "triangle in itself" in the world of sense, it is impossible for a human to encounter it. Of course, one has a chance to encounter the "image of a triangle" (A141) when he or she sees the pyramids in the Egyptian desert. However, if one does not know that pyramid's image signifies the analogy of "the triangle in itself," how he or she can apprehend "a triangle" through the pyramids' image? It is impossible. Therefore, we, while acknowledging that the "geometrical cognition" in regard to a triangle appears to be immediately evident, have to say that 1) the geometrical cognition cannot be grounded on "pure intuition" (A22) or "intuition a priori" (B137), but on the "intellectual intuition" (B307), 2) the object - "the triangle in itself" - is not given through sensibility but is given in mere "synthetic cognitions" (A301). The "intuition a priori" or the "a priori intuition," which, Kant seems to think, has nothing to do with "the object in itself" (A45), appear to be "data for possible experience" (A239) while pure intuition is homogeneous with "empirical intuition" (A22) from which "everything that belongs to sensation" (A22) is detached. Therefore, when Kant makes remarks in regard to pure intuition, a priori intuition and empirical intuition, saying, "We ordinarily distinguish quite well between that which is essentially attached to the intuition of appearances, and is valid for every human sense in general, and that which pertains to them only contingently because it is not valid for the relation of sensibility in general but only for a particular situation or organization of this or that sense. And thus one calls the first cognition one that represents the object in itself, but the second one only its appearance. This distinction, however, is only empirical" (A45), we partly agree with him, thinking that 1) "that which is essentially attached to the intuition of appearances" is commensurate with pure intuition, 2) a priori intuition appears to be homogeneous with pure intuition, i.e., "empirical intuition" from which "everything that belongs to sensation" is detached, 3) "that which pertains to them only contingently" is homogeneous with empirical intuition. Is "a priori intuition" distinct from pure intuition? We say, "yes, it is," or "no, it is not." Why? It is because we have to think that "pure intuition," which is "to be encountered in the mind a priori, wherein all of the manifold of appearances is intuited in certain relations" (A20), "occurs a priori, even without an actual object of the senses or sensation" (A21), while "a priori intuition," which is not encountered in the mind a priori, occurs a priori in conformity with the disappearance of an actual object of the senses or sensation. In other words, a priori intuition is homogeneous with a "pure concept, insofar as it has its origin in the understanding and in a pure image of sensibility" (YAMAMOTO 2018: 20-45), indicating that "sensation in itself" (B208) needs to be attached to a priori intuition. Therefore, a priori intuition should be considered to belong to "a pure category, in which abstraction is made from any condition of sensible intuition" (B304), which is necessarily accompanied by a pure image of sensibility (e.g., the extensive magnitude). Sensation in itself pertains to "an intensive magnitude, i.e., a degree of influence on sense" (B208). From this, it follows that it is possible to say that 1) since the sum total of "an object, as appearance, which is taken up into empirical consciousness through empirical intuition, is homogeneous with intensive magnitude" (YAMAMOTO 2017a: 19-37), an intensive magnitude - "a sum total of mere relations" (B321) - is correspondent to "intuition and the real" (B221) in perception, which "could be generated in accordance with rules of a mathematical synthesis" (B221) - "numerical magnitudes" (B221) - 2) "the intensive magnitude," i.e., "a sum total of mere relations" is parallel, in numerical magnitudes, to "the extensive magnitude," i.e., "the inner determinations of a substantia phaenomenon" (B321) in space-time, 3) therefore, the extensive magnitude (thereby the intensive magnitude) is homogeneous with a priori intuition to which sensation in itself is attached. Since the "degree of reality" (B210) is called "as cause a 'moment,' e.g., the moment of gravity" (B210), the intensive magnitude (thereby the extensive magnitude) is parallel to the moment of gravity, while the extensive magnitude (thereby the intensive magnitude) is to the converse moment of gravity. Since a priori intuition, which is not encountered in the mind a priori, has "qualitative relations" (B222) to "pure intuition," "wherein all of the manifold of appearances is intuited in certain relations," we can say that 1) all such things as " $a$ priori intuition which needs to be accompanied by sensation in itself", or "a pure concept which has its origin in the understanding and in a pure image of sensibility," or "a pure category, in which abstraction is made from any condition of sensible intuition" signify "pure schema of magnitude (quantitatis)" (B182) (e.g., the moment of gravity and the converse moment of gravity) - "number" 2) "number" is to pertain to "the domain" (RUSSELL 1971: 52-62) and to "the converse domain" at the same time, 3) the converse domain is commensurate with "a pure category, in which abstraction is 
made from any condition of sensible intuition" (B304). In contrast to "number," if "the triangle in itself," which is not in the world of sense, can be cognized through "a priori intuition," the "a priori intuition" which has nothing to do with "sensation in itself" is a mere "synthetic proposition" (A48) or a "singular proposition" (B205), though it might be enhanced to the possibility of pure a priori intuition or "pure a priori cognitions" (A119). Since a priori intuition needs to be accompanied by sensation in itself, it appears to be distinct from "pure intuition," from which "everything that belongs to sensation" (A22) is detached while a priori intuition and pure intuition appear to be homogeneous with "the noumenon in a positive sense" (B307) or "another kind of intuition than the sensible kind" (A254).

If it is shown that pure intuition is necessarily combined with a priori intuition, the "pure $a$ priori intuitions" (B73) or "intuitions themselves (which contain a manifold)" (B160) can be said to signify a "true correlate, i.e., the thing in itself" (A30). In order for pure a priori intuitions or intuitions themselves to be in accordance with "the thing in itself," this proposition must belong to "synthetic $a$ priori propositions" (B294), which rest on "synthetic a priori judgments" (A158) and "synthetic a priori cognitions." Our synthetic a priori propositions in regard to "pure a priori intuitions" (YAMAMOTO 2018: 20-45) states that "the conditions of the possibility of experience in general are at the same time conditions of the possibility of the objects of experience" (A158). In other words, "the conditions of the possibility of experience" and "conditions of the possibility of the objects of experience" are to be commensurate with "the condition of the possibility of the things themselves as objects of experience" (B258) - "a manifold of sensibility" (B102). Since an "a priori judgment" (B73) rests on "the intuition that corresponds to it" (B73), it "never extends beyond the objects of the senses and can hold only for objects of possible experience" (B73), indicating that an "a priori judgment" is homogeneous with "a perception itself" and "a manifold of sensibility." In view of the fact that the "synthetic a priori judgments," which extend beyond the objects of the senses, can be held for both objects of possible experience and objects of experience, we can say that "pure a priori intuitions," which rest on "a priori judgment," is the "synthetic a priori propositions" (B73), thereby "synthetic a priori judgments." Since "synthetic a priori judgments" rest on the "metaphysical deduction" (B159) in conjunction with "transcendental deduction" (B159) - on the "constitutive principles for the extension of our cognition to more objects than experience can give" (A671/B699) and the "regulative principles for the systematic unity of the manifold of empirical cognition" (A671/B699), namely "decomposition to infinity" (A526/B554) and "the infinite divisibility" (B206), "objects of possible experience," which a manifold of sensibility is to encounter in the world of sense, pertain to "the things themselves, which appear" (B324), e.g., a human or "a plant, an animal, the regular arrangement of the world's structure (presumably thus also the whole order of nature)" (B374A318). When Kant makes a discourse in regard to "empirical intuition," saying, "there is nothing to be encountered in it that pertains to anything in itself, then our transcendental distinction is lost, and we believe ourselves to cognize things in themselves" (A45), we agree with him in the belief that "a thing in itself which one thought through pure concepts of the understanding" (A525/B553), namely through "decomposition to infinity" and "the infinite divisibility," is homogeneous with "the a priori concepts of space and time" (B57), which signify "realitas noumenon," e.g., "a priori intuition" (B40) - the offshoot of "a faculty of synthesis a priori" (A123) - "the origin of which must really be sought in experience, out of whose abstracted relations imagination has made something that, to be sure, contains what is general in them" (B57). We believe that abstracted relations "that pertain to anything in itself" manifest through encountering nullity in space-time, i.e., "objects of possible experience." We rephrase "our transcendental distinction is lost" such that "the transcendental distinction between the 'domain' and the 'converse domain' (RUSSELL 1971: 52-62) is lost." Then, the loss of the "transcendental distinction" - the loss of "abstracted relations" - is tantamount to our cognizing "a thing in itself which one thought through pure concepts of the understanding." In regard to this issue, we have already made "synthetic a priori propositions," saying, "we think that the change of the accidents, namely ' $0 \rightarrow 1 \rightarrow i^{2}=-1 \rightarrow 0$ ' or ' $0 \rightarrow i^{2}=-1 \rightarrow 0$ ' signifies the synthetic $a$ priori propositions on account of the fact that 1) these propositions are 'valid only in relation to possible experience' (B228), and 2) they are to be proved successively through 'our apprehension of the manifold of appearance' (A182)... humans are to apprehend the manifold of appearance - nullity in space-time - upon encountering the disappearance of the manifold, namely death itself in regard to Homo sapiens. Since death itself is 'necessity,' among Homo sapiens, which 'is nothing other than the 
existence that is given by possibility itself' (B111), i.e., 'real possibility' (B302), the 'synthetic $a$ priori propositions' (B205), i.e., ' $0 \rightarrow 1 \rightarrow i^{2}=-1 \rightarrow 0$ ' or ' $0 \rightarrow i^{2}=-1 \rightarrow 0$ ' can be proved through a deduction of the possibility of 'possible experience,' i.e., a deduction of the possibility of death itself,..." (YAMAMOTO 2018: 20-45). It should be stressed that in regard to the change of the "accidents" (A186), the "synthetic a priori propositions," i.e., " $0 \rightarrow 1 \rightarrow i^{2}=-1 \rightarrow 0$ " or " $0 \rightarrow i^{2}=-1$ $\rightarrow 0$ " are "valid only in relation to possible experience, hence that they can be proved only through a deduction of the possibility" (B228-A185) of "possible empirical cognition" (A644/B672), since "accidents" are nothing other than particular ways for "the existence of the substance" (A186). Therefore, we have to say, in an opposite manner to what Kant refers to (A185), that "it is a wonder that the 'synthetic a priori propositions,' i.e., ' $0 \rightarrow 1 \rightarrow i^{2}=-1 \rightarrow 0$ ' or ' $0 \rightarrow i^{2}=-1 \rightarrow 0$ ' have, to be sure, grounded all possible experience, and can be proved through a deduction of the possibility of death itself."

If something exists whose cognition has never been proven through "experience itself" (A123), it is "death itself," though it is possible to cognize its "possibility itself" or its "real possibility" synthetically a priori in "the synthesis of apprehension" (A102) which is "inseparably combined with the synthesis of reproduction" (A102). The synthesis of apprehension or "the apprehension of the intuition" (A143) is to be evoked in accordance with exposure to a life-threatening peril - the real possibility of one's own demise. Since "apprehension is only a juxtaposition of the manifold of empirical intuition" (B219), if, in primordial times, there was something about which one could "anticipate experience precisely in what concerns its matter" (B209) in association with "apprehension," it was the "decomposition" (A525/B553) of one's feeder - a juxtaposition of the feeder's "experience precisely in what concerns its matter" (YAMAMOTO 2017a:19-37), implying that decomposition - "the divisibility of space" (A525/B553) - could be cognized synthetically $a$ priori prior to experiencing it, through the "reproductive synthesis of the imagination" (A102) and "the progressive decomposition" (A524/B552). Since decomposition concerns its matter, it pertains to "apprehension" - a juxtaposition of the manifold of empirical intuition, i.e., a juxtaposition of the moment of gravity and the converse moment of gravity.

We have to say that the synthesis of apprehension - the synthesis of pure intuition with a priori intuition - pertains to yielding "the pure concept of the understanding" (B224) - nullity in space-time, e.g., "decomposition to infinity." Therefore, the "possible judgment" - the "anticipation of perception" - which is to be evoked along with others" "decomposition," i.e., "a complete (and unconditioned) given condition" (A336), can be thought to be parallel to "the given judgment" (A330). In view of the facts that 1) the "anticipation of perception" in regard to decomposition to infinity - "the pure concept of the understanding" - is the given judgment, 2) it signifies "a representation of the necessary connection of the perceptions" (B219) - a perception itself - we dare to say that 1) a possible judgment in regard to the infinite divisibility, which a perception itself makes, is to be homogeneous with the given judgment in regard to decomposition to infinity, thereby its being equivalent to "an a priori judgment" (B73), 2) the synthesis of apprehension and the synthesis of reproduction are homogeneous with "the faculty of inferring, i.e., of judging mediately (through the subsumption of a condition of a possible judgment under the condition of something given)" (A330), 3) thus our singular proposition that the infinite divisibility - "the regressus in infinitum" (A519/B547) - is to be commensurate with decomposition to infinity - "a regressus in indefinitum" (A520/B548) - could be synthetic a priori propositions. An a priori judgment in regard to the infinite divisibility and the given judgment in regard to decomposition to infinity are the "ground for the possibility of a priori mathematical cognitions" (B57) - the ground for "pure mathematics" (A39) and "a priori mathematical doctrines in regard to real things" (B57). The "intuition and the real" (B221) in perception in regard to the infinite divisibility or decomposition to infinity is to be "generated in accordance with rules of a mathematical synthesis" (B221) - "numerical magnitudes" (B221). According to Kant, "perception is empirical consciousness, i.e., one in which there is at the same time sensation" (B207). Therefore, we say that an a priori judgment in regard to the infinite divisibility and the given judgment in regard to decomposition to infinity concern "the moment of gravity" - "a sum total of mere relations," i.e., the intensive magnitude - or the converse moment of gravity - a sum of abstracted relations, i.e., the extensive magnitude. Since "empirical cognition, however, is experience" (B165-B166), it becomes possible for us to say that " "the transcendental unity of the synthesis of the imagination' (A118), which comprises pure intuition, an a priori intuition and pure concept of the 
understanding - 'intuitions themselves' (B160) and 'perceptions themselves' (B219) - is 'the ground of the possibility of all cognitions' (A118), especially that of experience or of possible experience" (YAMAMOTO 2017b: 72-81).

We have shown that 1) "intuitions themselves" and "perceptions themselves" could "belong to one and the same universal experience" (A110) - "a priori principles of the possibility of experience" (B294) - 2) their combination, which "can be executed only by the subject itself" (B130) - "an act of its self-activity" (B130) - is to stand under "one and the same universal experience" (YAMAMOTO 2017a: 19-37, YAMAMOTO 2017b: 72-81). When Kant says, "as far as the laws of their combination are concerned, thus the possibility of as it were prescribing the law to nature and even making the latter possible, is to be explained" (B159-B160), we agree with him, thinking, in an opposite manner to what Kant says (B160), that "since the categories serve in this way, it becomes clear why everything that may ever come before our senses must stand under the laws that appear to arise $a$ priori from the understanding alone." What do "the categories" mean here? It is meant to be "number" which corresponds to the subject itself - an act of its self-activity - which is grounded on synthetic a priori judgments and synthetic a priori cognitions. In other words, the subject itself - an act of its self-activity, namely "number" - signifies "the combination of contradictorily opposed determinations in the existence of one and the same thing" (B291) and "of contradictorily opposed predicates (e.g., a thing's being in a place and the not-being of the very same thing in the same place) in one and the same object" (B48), which is to be commensurate with the metaphysical path, i.e., "synthesis speciosa" (B151) and the "transcendental path" (A87), i.e., "combination of the understanding (synthesis intellectualis)" (B151). This is "rules of a mathematical synthesis." We think that the synthesis speciosa, which pertains to "the identity" (B222) of "two qualitative relations" (B222) - the "identity of itself" (A108), i.e., "the ground of the identity of apperception itself" (B134) - and synthesis intellectualis, which pertains to "one," i.e., the identity of two quantitative relations "the identity of the substratum" (A186) - signifies the "combination a priori" (B131). The identity of "two qualitative relations" and of "two quantitative relations" - the identity of itself and the identity of the substratum - necessarily and universally stands under "abstracted relations" (B57) and "mere relations." "A sum total of mere relations," i.e., "an intensive magnitude" (B208) and a sum of "abstracted relations," i.e., the "extensive magnitude" (A173) signify "the absolute whole of magnitude (the world-whole)" (A483) - the "entire universe" (A274). Since the "intensive magnitude" - "a sum total of mere relations" - cannot be determined in it, "we must progress further to this condition" (A520/B548), indicating that the "intensive magnitude" is to be determined in a regressus in indefinitum. However, in view of the fact that a regressus in indefinitum - decomposition to infinity - and the regressus in infinitum - the infinite divisibility - are always exactly the same since both stand under the "supreme cause of the world" (A688/B716) - "the objects of nature itself," i.e., space-time itself - it becomes possible to think that "the identity of the substratum," i.e., the "extensive magnitude" can be determined in the regressus in infinitum.

When Kant states his belief that "the cognition itself a priori in intuition (as far as their form is concerned)" (A88) is attained through "a priori intuition," this cognition does not pertain to pure intuition nor empirical intuition. In view of the fact that "synthetic cognitions" do not correspond to "synthetic a priori cognitions," Kant's "cognition itself a priori in intuition" seems to belong among mere "synthetic cognitions" such as cognizing the triangle in itself - "all geometrical cognition" (B120) - which he calls "principles absolutely" (B358). If "the understanding cannot yield synthetic cognitions from concepts at all" (A301-B358), "reason" must yield them through "self-cognition" (B294). The "self-cognition" of "the triangle in itself," which can be neither "synthetic a priori propositions" (B294) nor "universal propositions a priori" (B357) belongs to mere "synthetic cognitions," though it can be enhanced to a proposition which is valid "only for a particular situation or organization of this or that sense." Kant confesses that he himself feels concern about the "synthetic cognitions," saying, "Since I am now to give a definition of this supreme faculty of cognition, I find myself in some embarrassment" (A299). What does "this supreme faculty of cognition" mean? It is meant to be "geometry" (B206) - "the rules of the infinite divisibility of lines or angles" (B206), e.g., the schemata of "the shadows" (YAMAMOTO 2018: 20-45) - which is, Kant seems to think, grounded in "the first faculty" (A299) of reason - "a merely formal, i.e., logical use, where reason abstracts from all content of cognition" (A299) and "the second faculty, which itself generates concepts" (A299) - "a transcendental faculty" (B356). Here, we have to note that 1) in the 
merely formal use of the faculty of reason, "reason abstracts from all content of cognition," 2) therefore, "the first faculty" of reason appears to be homogeneous with "the second part of the transcendental logic" (B88), i.e., "transcendental dialectic" (B88), while "the first faculty has obviously long since been defined by the logicians as that of drawing inferences mediately (as distinct from immediate inferences, consequentis immediatis)" (A299). The product of the "transcendental dialectic" is commensurate with the "mathematical axioms" (A300) such as Peano axioms or Euclid's Common Notions (YAMAMOTO 2017c: 57-70, YAMAMOTO 2017d: 19-29, YAMAMOTO 2018: 20-45). We think that there is a serious problem in Peano axioms or Euclid's Common Notions (YAMAMOTO 2017c: 57-70, YAMAMOTO 2017d: 19-29, YAMAMOTO 2018: 20-45). They appear to be irrational. Furthermore, the "propositions of numerical relation" (B205) such as "that $7+$ $5=12$ " (B205) is so self-evident that the "mathematical axioms" (A300) in regard to numerical relation must not be a mere "synthetic proposition," thereby being a "singular proposition," but must be "analytic proposition" (B205). Even if Peano axioms or Euclid's Common Notions or mathematical axioms appear to be the "universal proposition" (A300), "it is not therefore itself a principle" (A300). Why is it not? It is because mathematical axioms are "taken from experience (by induction)" (A300), which indicates that Peano axioms or Euclid's Common Notions or mathematical axioms have been made for the use of mathematicians inside the cave (YAMAMOTO 2018: 20-45). We have already clarified that though "inductive numbers" (RUSSELL 1971: 29-41) or Peano axioms or Euclid's Common Notions cannot be the "synthetic a priori principles" (B305), they are to be enhanced to provisional "principles" or "the copula" (B142) through the schemata of "the shadows of the artificial objects," which can be thought (by the people outside the cave) to arise in "logical reflection" (B318) or in "mere comparison (comparatio)" (B318) with the physical world (YAMAMOTO 2018: 20-45). When the number is assigned to mathematicians inside the cave, it is to "serve as the major premise in a syllogism" (A300) inside the cave. On the contrary, if "universal proposition" is taken from experience (by deduction), it can be a true "principle" or a "synthetic $a$ priori principle." We think that, by means of "producing a chain of deductions with no link missing, such that no step in it is taken which does not conform to some one of a small number of principles of inference recognized as purely logical" (FREGE 1980: 99-119), "inductive numbers" can be shown, through the "transcendental synthesis of the imagination" (B152) - "synthesis of intuitions, in accordance with the categories" (B152) - to belong to the category or the categories, e.g., "intuitions themselves" or "a perception itself" (A180) - perceptions themselves. Here, we have to take note of the facts that 1) "the transcendental synthesis of the imagination," which pertains to the synthesis of an a priori intuition (a pure category) with pure intuition (a priori categories), rests on "the synthesis of apprehension" (A99) and "the synthesis of representations" (A155) - synthesis speciosa and synthesis intellectualis - 2) synthesis speciosa and synthesis intellectualis have a one-one relation, 3) since synthesis speciosa and synthesis intellectualis - "intuitions themselves" and "perceptions themselves" - can have a cognition of oneself "as I am" (B158) and "as I appear to myself" (B158), synthesis speciosa and synthesis intellectualis in virtue of "the cognition itself a priori in intuition" belong among "a synthetic a priori concept" (A220), 4) "the cognition itself a priori in intuition" signifies "the regress of the decomposing synthesis" (A505/B533) - "the regressive synthesis itself" (A505/B533) and "the regress itself" (A514/B542), i.e., "the progressive decomposition." "The cognition itself a priori in intuition" corresponds to the "transcendental unity of self-consciousness" (B132), which is the "necessary synthetic unity" (A79) for "the synthetic principles or formulas" (A240). Therefore, we can think that "the transcendental synthesis of the imagination" along with "the synthesis of apprehension" and "the synthesis of representations" - "the regress of the decomposing synthesis" - pertains to yielding the "pure schema of magnitude (quantitatis)," i.e., "number" or "the synthetic principles or formulas," i.e., $\left\{i^{2} \cdot e^{i \pi}\right\}=$ "1." Furthermore, we say that 1$)$ "the regress of the decomposing synthesis" - the regressive synthesis itself, namely "the mere continuation of productive synthesis of a certain kind" (B212) and "the regress itself," namely "the repetition of an ever-ceasing synthesis" (B212) - is "the true and sole conditions" (A146) for providing the transcendental synthesis of the imagination "with a relation to objects, thus with significance" $(A 146), 2)$ the regressive synthesis itself pertains to "the schemata of the concepts of pure understanding" (B185A146), i.e., the schemata of the category and the categories while "the regress itself" to the "schema of the pure concept of the understanding" (B224), i.e., the schema of the category, 3) the regressive synthesis itself and the regress itself serve to subject appearances to "the same inner determinations (qualitas et quantitas)" (B319), i.e., transcendental space-time-determination, and "to general rules of 
synthesis through grounds of an a priori necessary unity" (A146), thereby making them fit for "a thoroughgoing connection in one experience (A146). Therefore, we can think that the transcendental synthesis of the imagination along with synthesis speciosa and synthesis intellectualis is parallel to the "pure synthesis of the imagination" (A118) along with the "pure synthesis of apprehension" (A100) and "pure synthesis of representations" (B104) - pure mathematics - indicating that "the regressive synthesis itself" is homogeneous with "the regress itself." What do the "schema of the pure concept of the understanding" and the "schemata of the concepts of pure understanding" mean? They mean the schema and the schemata of "transcendental reflection" (B325) and "logical reflection" (B318) in regard to "the object in accordance with what constitutes its concepts (magnitude, reality)" (B325) the "pure schema of magnitude (quantitatis)," namely "number." Therefore, "number" in virtue of "universal propositions a priori" or "principles" (B357) - the "transcendental principle of the mathematics" (B206) in regard to appearances themselves - can "serve as the major premise in a syllogism" (YAMAMOTO 2016: 87-100, YAMAMOTO 2018: 20-45). Since the transcendental principle of the mathematics in regard to appearances themselves, such as the category and the categories or "possibility itself" and "possibility," is not "the deception of substituting the logical possibility of the concept (since it does not contradict itself) for the transcendental possibility of things (where an object corresponds to the concept)" (B302), it can be called "first-order logic." Here we have to take note of the fact that "the propositions of geometry" (B64), which appear to be cognized synthetically a priori, such as "that there can be only one straight line between any two points)" (A300) cannot be the "universal propositions a priori" since it is not possible for a human to encounter the triangle in itself in the world of sense. We dare to say that they belong among mere synthetic proposition.

Next, we have to discuss about "the second faculty" of reason. We would like to say that 1) "the second faculty, which itself generates concepts" - a transcendental faculty of reason - appears to be homogeneous with "general logic" (B102), which "abstracts from all content of cognition" (B102), 2) general logic is distinct from the first faculty of reason on account of the fact that in the first faculty of reason, "reason abstracts from all content of cognition" (A299), thereby being "always transcendent" (A327), while in general logic, the transcendental faculty of reason could make use of the abstraction from all content of cognition, in order for it to transform representations "given to it from elsewhere" (B102) into "concepts analytically" (B102), thereby being "synthetic and immediately certain (indemonstrabilia)" (A164), 3) in order for reason to properly employ general logic, it absolutely needs "the part of transcendental logic" (B87) - the "transcendental analytic" (B87) - which "has a manifold of sensibility that lies before it a priori, which the transcendental aesthetic has offered to it" (B102-A77). It becomes possible to think that if general logic runs along with the transcendental analytic, the "propositions of numerical relation" such as "that $7+5=12$ " could be self-evident, indicating that the "pure a priori cognitions" (A119) - mathematical axioms - are in accordance with metaphysical axioms (YAMAMOTO 2017c: 57-70, YAMAMOTO 2017d: 19-29, YAMAMOTO 2018: 20-45). In regard to the issue of the transcendental logic and general logic, we have already said that "it is the 'business treated by general logic' (B104) that analytically brings different representations 'under one concept' (B104). General logic happens to be merely a thought 'without any content,' which might be completely empty, provided representations are not given from elsewhere...Here, it is important to notice that representations treated by transcendental logic are not the same as those treated by general logic. The former is supposed to bring 'the pure synthesis of representations' (B104) under concepts, through cognizing 'a manifold of sensibility that lies before it a priori' (B102), while the latter is to analytically bring different representations, which are given elsewhere, 'under one concept' (B104)" (YAMAMOTO 2016: 87-100). Apparently, general logic needs the part of transcendental logic - the transcendental analytic - prior to itself or in conjunction with itself. It is impossible to separate general logic from the transcendental analytic on account of the fact that representations must be given to general logic through cognizing "a manifold of sensibility that lies before it a priori" - "the progressive decomposition," i.e., "the regress itself." Since general logic absolutely needs the transcendental analytic, we have already said, "First the procedure of general logic is followed since it seems to be associated with the law of nature. General logic enables us to bring different representations under one concept analytically if the representations are given from elsewhere, where the law of nature prevails" (YAMAMOTO 2016: 87-100). In this context, it becomes possible for us to say that 1) general logic could be the combination of the part of transcendental logic, i.e., the transcendental analytic and a transcendental faculty of reason, i.e., the 
second faculty, which itself generates concepts by making use of "the relations of things in abstracto" (B342), 2) the product of general logic, i.e., "the transcendental product of the imagination" (A142) signifies "universal propositions a priori" or "principles" - metaphysical axioms (e.g., a human or Homo sapiens) - which can "serve as the major premise in a syllogism," 3) therefore, "the transcendental product of the imagination, which is to correspond to "the universal rule (major premise, major)" would be "first-order logic" (SNAPPER 1979: 207-216, HODEL 2013: 167-218) "immediate inferences, consequentis immediatis" (A299). The "synthetic judgments" (A154), i.e., "immediate inferences, consequentis immediatis" are to be correspondent to an a priori judgment in regard to the infinite divisibility and the given judgment in regard to decomposition to infinity - the synthetic a priori judgments in regard to "the moment of gravity," i.e., "a sum total of mere relations." Here we have to stress that "first-order logic" has nothing to do with transcendental dialectic. It signifies "the categorical syllogisms, whose major premise, as a principle, states the relation of a predicate to a subject" (A406-B433), i.e., the relation of "contradictorily opposed predicates in one and the same object" to "contradictorily opposed determinations in the existence of one and the same thing." This means that the identity of "two qualitative relations" - the identity of itself - could be correspondent to the identity of two quantitative relations - the identity of the substratum. Thus, first-order logic - "a recognition-judgment" (FREGE 1980: 99-119) - comprises "a judgment-content which can be transformed into an identity" (FREGE 1980: 99-119) - indicating that it is to be commensurate with "the causality of an alteration" (B213). We can say that first-order logic could be "a universal law - even of the possibility of all experience - that everything that happens must have a cause, and hence that the causality of the cause, as itself having happened or arisen, must in turn have a cause" (A533/B561). First-order logic, which is grounded in "sensation in itself," "possible experience," "transcendental deduction" along with "metaphysical deduction" (YAMAMOTO 2016: 87-100, YAMAMOTO 2017a: 19-37, YAMAMOTO 2017b: 72-81, YAMAMOTO 2017c: 57-70, YAMAMOTO 2017d: 19-29, YAMAMOTO 2018: 20-45) would lead us to "a formula of PM with exactly one free variable, which is of the type of the natural numbers (class of classes)" (GÖDEL 2004: 5-38).

\section{A SOMETHING $=\mathbf{X}$, A THIRD THING, GENERAL LOGIC AND NUMbERS}

Since the "transcendental analytic" "has a manifold of sensibility that lies before it a priori," "a something $=X$ " (A250) could arise through "the progressive decomposition," i.e., "the regress itself." If one succeeds in cognizing "a something $=X$ " synthetically a priori by means of the "anticipation of perception" (B217) and "mere perception (sensation and thus reality)" (B212), mobilizing sensibility, understanding and reason, it becomes possible to say that the anticipation of perception in virtue of a perception itself - the given judgment in regard to decomposition to infinity - could correspond to "an a priori judgment" in regard to the infinite divisibility. The given judgment in regard to decomposition to infinity pertains to a something $=X$ while an a priori judgment in regard to the infinite divisibility pertains to "a third thing, which must stand in homogeneity with the category on the one hand and the appearance on the other, and make possible the application of the former to the latter" (A138/B177). A something $=X$ and a third thing are the ground of the "synthetic a priori cognitions" in regard to "appearances themselves," i.e., "things in themselves." We think that the schema of a something $=X$ and the schemata of a third thing are nothing other than first-order logic. Prior to attaining synthetic a priori cognitions, it is absolutely necessary that 1 ) one has to encounter a something $=X$ in "experience itself" or in "possible experience," 2) one must comprise the elements of a something $=X$ or a third thing, 3 ) a something $=X$, namely "transcendental object" (A250) and a "third thing, namely a pure object" (A157) - the "transcendental schema" (A138/B177) - are composed of the same elements as a manifold of sensibility that lies before it a priori, 4) one determines a cognition, which "starts from the senses, goes from there to the understanding, and ends with reason" (B355). We would like to put it another way, saying that 1) "the second faculty, which itself generates concepts" must be necessarily accompanied by all content of cognition - the schema of a something $=X$ and the schemata of a third thing -2 ) a something $=X$ is to be necessarily drawn from the transcendental analytic, 3) all content of cognition must stand under the law of nature. This means that the law of nature is homogeneous with all content of cognition - first-order logic. When "representations" are given to general logic "from elsewhere" (B102), namely from the domain, the schema of a something $=X-$ the synthetic $a$ priori cognitions - signifies "generally valid judgments (judicia communia)" (A71); which speaks that "the predicate therefore holds of that concept without 
exception, just as if the latter were a generally valid concept with a domain with the predicate applying to the whole of what is signified" (A71). Thus when a reason properly employs "the second faculty, which itself generates concepts," a merely formal, i.e., logical use of the faculty of reason the formal logic - will be enough insofar as "all content of cognition" is provided "from elsewhere," in other words, insofar as representations will be given to it "by means of a schema of that unity" (A697/B725) - the schema of a something $=X$ and the schemata of a third thing. Here, we would like to say that "a schema of that unity" - the schema of a something $=X$ and the schemata of a third thing - is commensurate with our "metaphysical axioms" (thereby mathematical axioms) (YAMAMOTO 2017c: 57-70, YAMAMOTO 2017d: 19-29), which could be first-order logic. However, there arises a serious concern about this discourse, which claims that all content of cognition or representations are to be given prior to synthetic a priori cognitions. Here, one thing is asserted: all content of cognition or representations must come together from elsewhere. "All content" is to come from the law of nature, while "representations" are to come from the cognition of "all content," namely from the cognition of the law of nature, indicating that "all content," "the law of nature," "representations" and "cognition" must arise from itself. This appears to be a tautology.

Since a something $=X$ is to arise from the "transcendental analytic," the element from which it is to arise resides in a manifold of sensibility or "the manifold of appearance" (A182) - filled spaceelapsing time or empty space-nullified time. In regard to the "element," we have already explicated, saying, "Since 'appearances themselves are nothing but sensible representations' (A104), the elements of which an object consists should be the sensible representations, which are equivalent to the 'pure synthesis of representations' (B104). What are the elements? They are nullity, in just the same way as empty space-nullified time" (YAMAMOTO 2016: 87-100). Since empty space-nullified time is commensurate with nullity in space-time, i.e., space-time itself (YAMAMOTO 2016: 87-100, YAMAMOTO 2017a: 19-37), we would say that the "element," from which the schema of a something $=X$ is to arise in the image of "a parte priori without bounds" (B445-A418) or from which the schemata of a third thing is to arise in the image of "the matter of all possibility" (B322), is "a manifold of sensibility that lies before us a priori" - "what is unconditioned in every relation" (A326), i.e., decomposition to infinity. The field from which a something $=X$ is to arise is the same as that from which a third thing is to arise. Since a something $=X$ and a third thing are to arise in perception as "beings of understanding (noumena)" (B306) through rules of a mathematical synthesis, the anticipation of perception as a perception itself, which pertains to the schema of a something $=X$ and the schemata of a third thing, belongs to synthetic a priori cognitions or the given judgment. We put it another way, saying that a "synthetic a priori cognition" in regard to the schema of a something $=X$ or the schemata of a third thing is grounded in the "object of all possible experience, i.e., nature" (A114).

First-order logic, as the "universal propositions a priori" or the "synthetic a priori propositions" in regard to Homo sapiens, signifies "the universal rule (major premise, major)," which could be the ground of the synthetic a priori judgments. We think that in order for a human to attain the status of major premise, one absolutely needs three things: "sensibility itself" (A20), e.g. the light itself or an appearance, "the understanding as the faculty of rules" (B356), e.g., the moment of gravity, and "the faculty of principles" (B356), i.e., "number" and "numerical formulas." It is critically important for us to acknowledge that, prior to the understanding and reason, humans must encounter the "objects of nature itself," which could evoke a chain of events, leading them to attain "the universal propositions a priori" - synthetic a priori propositions in regard to "object of all possible experience." We have to stress that the "supreme faculty of cognition" (A299) cannot be separated from the possibility itself of possible experience, the cognition of which rests on pure intuition, a priori intuition and sensation in itself. Otherwise, the "synthetic cognitions" (A301) or the synthetic a priori cognitions would be nothing but "a mere figment of the brain" (A157). Therefore, when Kant makes a discourse in regard to "experience" and "cognition," saying, "since experience, as empirical synthesis, is in its possibility the only kind of cognition that gives all other synthesis reality, as a priori cognition it also possesses truth (agreement with the object) only insofar as it contains nothing more than what is necessary for the synthetic unity of experience in general. The supreme principle of all synthetic judgments is, therefore: Every object stands under the necessary conditions of the synthetic unity of the manifold of intuition in a possible experience" (A157-B197-A158), we agree with him, thinking that 1) the propositions of geometry, as mere synthetic proposition, are distinct from other synthetic propositions 
such as the schema of a something $=X$ and the schemata of a third thing, 2) the schema of a something $=X$, i.e., "the regress itself" and the schemata of a third thing, i.e., "the regressive synthesis itself," signify the "synthetic unity of experience" (B197-A158). Since a human - "pure intuition (in mathematics) or the conditions of a possible experience" (A301) - is nothing but the "synthetic unity of experience," we - a schema of that unity - have to say that the schema of a something $=X$, i.e., "the regress itself" and the schemata of a third thing, i.e., "the regressive synthesis itself" could be enhanced to the "principles absolutely" (B358) - "the supreme principle of all synthetic judgments," indicating that "no a priori cognition is possible for us except solely of objects of possible experience" (B166). A human "contains nothing more than what is necessary for the synthetic unity of experience in general." Therefore, a human or Homo sapiens can attain "the supreme principle of all synthetic judgments," which corresponds to a priori cognition of objects of possible experience, i.e., $a$ priori cognition of appearances themselves. This is tantamount to saying that a priori cognition in regard to "the infinite divisibility" or "decomposition to infinity" of "the things themselves, which appear" - a priori cognition in regard to the possibility itself of possible experience and the possibility of experience itself, namely "the regress itself" and "the regressive synthesis itself" - is possible for us. We think that 1) a priori cognition signifies the "necessity" (B111), i.e., "the existence that is given by possibility itself" (B111), 2) since "experience is a cognition of objects through perception" (B219) - a cognition of the possibility of experience itself or of the possibility itself of possible experience - "the determination of the existence of objects" (B219) in space-time "can only come about... only through a priori connecting concepts" (B219) - a something $=X$ and a third thing -3 ) a something $=X$ signifies the necessary connection of objects through perception, which "is or can become evident in the perceptions themselves" (B219), 4) since a third thing must "stand in homogeneity with the category on the one hand and the appearance on the other" (A138/B177), the "mediating representation" (A138/B177) is homogeneous with the transcendental schema, namely synthesis speciosa and synthesis intellectuals. Since the synthesis speciosa and the synthesis intellectualis, which are to pertain to the "possibility itself of death," namely death itself, take place only among Homo sapiens through "pure intuition affected by the sensation of nullity" (YAMAMOTO 2017a: 19-37), we can say that the pure category (e.g., a priori intuition) and a priori categories (e.g., the pure intuitions) "possesses truth (agreement with the object) only insofar as it contains nothing more than what is necessary for the synthetic unity of experience in general" (B197A158) - the schema of something $=X$ and the schemata of a third thing - which is commensurate with first-order logic. When Kant makes a discourse in regard to intuition, saying, "the sensible faculty of intuition is really only a receptivity for being affected in a certain way with representations, whose relation to one another is a pure intuition of space and time" (A494), we agree with him. "A certain way," through which the pure intuition (thereby a priori intuition) and sensation in itself are affected with representations, is an "instantaneous and simultaneous reciprocity-converse," e.g., "the regress of the decomposing synthesis" or "the regressive synthesis itself." This is to take place synthetically $a$ priori in "intuitions themselves (which contain a manifold)" and perceptions themselves - the transcendental unity of the synthesis of the imagination. From this it becomes possible to say that 1) the "possible empirical concept" (A489/B517) is shot out from "objects in themselves in which the absolutely unconditioned might possibly occur"(A508/B536) - "appearances in the field of experience" (A186) - while the "empirical concepts" (A47) - concepts or intuition given a posteriori - is shot out from "objects in themselves" in which the absolutely unconditioned has already occurred - "the field of possible empirical cognition" (A644/B672) or "the field of possible experience" (A248-B305) - 2) in case of objects in themselves, which manifest as the possibility itself of possible experience or the possibility of experience itself," "the field of possible experience" is to correspond to "objects of possible experience," 3) "all possible empirical cognition" (A680/B708) in regard to the possible empirical concept necessarily occurs inside "the bounds of experience" (A462/B490) while that in regard to the empirical concepts necessarily occurs inside "the bounds of possible experience" (A644/B672). Here, we have to take note of the facts that 1) while "all propositions of geometry" (A47) or "all geometrical cognition" (B120) is supposed, by Kant, to be grounded on "intuition $a$ priori," there is no "triangle in itself" in the world of sense, 2) "all geometrical cognition" could be parallel to "a system of the epigenesis of pure reason" (B167), indicating that the "geometrical cognition" is not grounded on the "pure intuitions" and sensation in itself - "elements of cognition that are to be encountered in us a priori" (B166). We say that in regard to "the field of possible experience," i.e., nullity in space-time, "all of our cognitions, however, lie in the entirety of all 
possible experience" (A146), thereby being "transcendental truth, which precedes all empirical truth and make it possible" (A146), while "all geometrical cognition" in regard to "triangle in itself" could correspond to "a merely logical principle" (B192) - "the principle of contradiction" (B192) - which appears to be parallel to the schemata of the entire universe.

If we wish to attain the "assertion" in regard to a something $=X$ or a third thing, it is crucial for us to attain "the empirical reality" (B52) of space-time, i.e., "objective validity in regard to all objects that may ever be given to our senses" (B52). Furthermore, "since our intuition is always sensible" (B52), the problem that "no object can ever be given to us in experience that would not belong under the condition" (B52) of space-time itself has to be solved. In regard to these issues, we have already explicated (YAMAMOTO 2016: 87-100, YAMAMOTO 2017a: 19-37) as follows: 1) the empirical reality in regard to humans themselves has two phases; an "appearance itself" (B53), i.e., a human in a filled space-elapsing time and a converse "appearance itself," i.e., a human in nullity in space-time, 2) one can encounter the latter in another's "disappearance itself," which is commensurate with another's "experience precisely in what concerns its matter." Since a human is to encounter another's "disappearance itself" in the world of sense, one can cognize nullity in space-time through empirical intuition - through pure intuition affected by the sensation of nullity. Therefore, if a human, who cognizes nullity in space-time upon encountering all human's disappearance itself ("all" insofar as one can encounter them), makes "a singular proposition" that the particular "allness" - "particular cognitions" (A647/B675) - could belong to "pure a priori cognitions" which are to be subsumed under nullity in space-time, i.e., "in which abstraction is made from any condition of sensible intuition" (B304), we can think that the "particular cognitions," which take "the conditioned in the transcendental signification of a pure category" (A499), namely in the possibility itself of possible experience, is equivalent to the "major premise" of categorical syllogism on account of the facts that 1) the particular "allness" or the "particular determinations" (B279) - the "particular laws" (B165) are grounded in "a pure category," i.e., a priori intuition, 2) the particular allness or the particular laws can be enhanced to the universal "allness" - "universal cognition" (B357) - by means of the function of reason, which employs "general logic" (B104) wherein "different representations are brought under one concept analytically" (B104). The "universal cognition" corresponds to "the absolute unity of the thinking subject" (A335). Thus, the singular proposition is enhanced to the universal propositions $a$ priori. Since the "universal cognition" of death itself as the possibility itself of possible experience or the possibility of experience itself - "a priori cognition" (A157-B197) - possesses "truth (agreement with the object)," universal propositions a priori in regard to a priori cognition become the "principles," which could be conveyed throughout "the possibility of the category of community" (B292), namely human society. While the "principles" - number and numerical formulas - appear to be correspondent to "pure cognition" (B87), they rest on "cognizing a pure category," i.e., cognizing nullity in space-time. This is "the greatest possible manifoldness" (A700/B728), which is "to be encountered everywhere to infinity" (A700/B728). Therefore, we dare to say that 1) nullity in spacetime, i.e., space-time itself signifies a pure category - "nothing" (A517/B545) or "the void" (A517/B545) - which is to bound "appearance," namely a priori categories, 2) "a bounding of appearance by nothing, or by the void" (A517/B545) makes it possible for the appearance, to progress to infinity - "the progressive decomposition" - "by dismantling the parts to infinity" (A526/B554), which is equivalent to "a regress to infinity" (A518/B546), 3) the intensive magnitude, i.e., "a sum total of mere relations" (B321) or "the inner determinations of a substantia phaenomenon" (B321) in space-time signifies the moment of gravity, 4) a pure category and a priori categories manifest in "an indeterminately continued regress (in indefinitum)" (A518/546), i.e., the regressive synthesis itself or in "the regressus in infinitum," i.e., "the regress itself," as the extensive magnitude or the intensive magnitude, 5) thus "the cognition itself a priori in intuition," which signifies the intensive magnitude (i.e., $\left\{i^{2} \cdot e^{i \pi}\right\}=$ "1") and the extensive magnitude (i.e., a median class having $\aleph_{0}$ terms, i.e., $e^{i \pi}=-1=$ $i^{2}$ ), manifests through the "instantaneous and simultaneous reciprocity-converse" in such a manner as: "the regressus in infinitum" vs. "an indeterminately continued regress (in indefinitum)" or the regress itself vs. the regressive synthesis itself or the intensive magnitude vs. the extensive magnitude.

While death itself has never been proven through experiencing it, the possibility itself of "decomposition," which precisely "concerns its matter," could be cognized through "a rule for seeking it in experience and a mark for discovering it there" (A180), namely through the "synthesis of apprehension" (A99), i.e., "unity of intuition" (A99) and "the rule in accordance with which unity of 
experience is to arise from perceptions" (A180) - the "possibility of experience itself." In other words, the "analogy of experience" (A180) in regard to "objects in themselves in which the absolutely unconditioned might possibly occur" (A508/B536) pertains to "a priori principles of the possibility of experience" and the "reality combined with negation" (B111). The "mathematical principles, which are constitutive" (B223) and regulative, is "the way in which something is apprehended in appearance" (B221), which is "determined a priori so that the rule of its synthesis at the same time yields this intuition a priori in every empirical example" (B221). Since "this intuition a priori in every empirical example" is to correspond to the moment of gravity, the converse moment of gravity signifies the law of nature in regard to a human or an appearance or Homo sapiens. We say, in regard to "principles," that if it is cognized that "there is nothing to be encountered in it that pertains to anything in itself" (A45), the "transcendental distinction is lost, and we believe ourselves to cognize things in themselves" (A45) in "principles." It is tantamount to saying that "if there is nullity in spacetime to be encountered in experience itself, the transcendental distinction is lost, and we cognize things in themselves." Only in this way - through "that the a priori cognition be entirely pure" (A14/B28) - we can attain the truths; whatever is contained in a general concept is also contained in the particular ones that stand under it, and "the latter are particular concepts precisely because they contain more than is thought in general concept" (A281). We have already said that "though "the understanding cannot yield synthetic cognitions from concepts at all' (A301-B358), it can yield them from the comprehension of the law of nature, namely death itself, as possible experience. Thus, we clarified 'the part of transcendental logic' (B87), namely 'the transcendental analytic,' expounding 'the elements of the pure cognition of the understanding and the principles without which no object can be thought at all' (B87)" (YAMAMOTO 2016: 87-100). This means that we have found through "possibility itself" of "possible experience" - through "a priori principles of the possibility of experience" - that, "with regard to the pure intuitions as well as the pure concepts of the understanding, there are elements of cognition that are to be encountered in us a priori" (B166). The "elements of cognition that are to be encountered in us a priori" correspond to the pure intuitions ( $a$ priori categories), i.e., "objects in themselves in which the absolutely unconditioned might possibly occur" (A508/B536), which manifest in the moment of gravity as "number."

\section{First-Order logic, A Human, Homo Sapiens, the Category and One}

Since "general logic," which could be the combination of the "transcendental aesthetic" (B102-A77), the "transcendental analytic," and "the second faculty, which itself generates concepts," leads us to "synthetic a priori cognitions" - the "ground for the possibility of a priori mathematical cognitions" (B57) - we would make "synthetic a priori judgments" (A158) or "synthetic a priori propositions," in accord with what Kant says (A158), that "every object stands under the necessary conditions (the infinite divisibility and decomposition to infinity) of the synthetic unity $\left(\left\{i^{2} \cdot e^{i \pi}\right\}=\right.$ "1") of the manifold of intuition $\left(e^{i \pi}=-1\right.$ or $\left.i^{2}=-1\right)$ in a possible experience $\left(e^{i \pi}=-1=i^{2}\right)$." Humans absolutely need "general logic," i.e., "pure mathematics," which is to relate the "conditions of a priori intuition, the synthesis of the imagination, and its necessary unity in a transcendental apperception to a possible cognition of experience in general" (A158). Since the product of "general logic" belongs to the "synthetic a priori propositions" which are equivalent to the given judgment and an a priori judgment - "immediate inferences, consequentis immediatis" (A299) - it can "serve as the major premise in a syllogism," which is to be called "axioms" (A165), namely "first-order logic." While Kant calls it "applied (though still general) logic" (A54), we call it "first-order logic," which, we think, is commensurate with the cardinality in the domain; " $0 \rightarrow 1 \rightarrow i^{2}=-1 \rightarrow 0$ " or " $0 \rightarrow i^{2}=-1 \rightarrow 0$ " (YAMAMOTO 2017c: 57-70, YAMAMOTO 2017d: 19-29, YAMAMOTO 2018: 20-45). Seeing that it would lead us to "a formula of PM with exactly one free variable, which is of the type of the natural numbers (class of classes)" (GÖDEL 2004: 5-38), we think that the problems of Gödel's "incompleteness of every formalism" (GÖDEL 2004: 84-88) and Zermelo's axiom (RUSSELL 1971: 117-130) will be solved. Why do we try to solve these problems? Before answering this question, we have to acknowledge two things; 1) mathematics is enormously useful in the natural sciences, which is said to be "something bordering on the mysterious" (WIGNER 1960: 1-14), 2) physics cannot operate without mathematics, to the extent that "what is mathematics?" could be correspondent to "what is physics?" (WIGNER 1960: 1-14). Therefore, when we hear something baffling in regard to "numbers" from Frege, who says, "We have no doubt grown used to treating the numbers in many contexts as all of the same sort, but that is only because we know a set of general propositions which 
hold for all numbers... however, we must put ourselves in the position that none of these has yet been discovered" (FREGE 1980: 1-24), we feel concern about a solid foundation of mathematics. If mathematics is not grounded on the solid foundation, it might evaporate into emptiness, bringing physics together into the abyss of emptiness. It would be a total disaster for humans. Therefore, it is requested to find a solid foundation of mathematics, in order for it to be able to sustain the "theories of physics" and itself. Where does the clue to a solution of the problem reside. Frege shows us a direction to proceed, saying, "with the numbers we have difficulty in finding even a single common property which has not actually to be first proved common" (FREGE 1980: 1-24). What does "a single common property which has not actually to be first proved common" mean? We think that it is "first-order logic."

Why has not "a single common property" been first proved common so far? We think that there are three reasons. First, when Euclid stated, as "definitions" of geometry, "a point" and "a line" in nullity in space-time, saying that "a point is that which has no part" and "a line is breadthless length," it spawns "boundary" in nullity in space-time, leading ancient Greeks to think that "a figure is that which is contained by any boundary or boundaries" (EUCLID 2002: 1-36). This is the beginning of the conundrum in mathematics. In regard to this issue, we have already made a discourse, saying, "Natural numbers in primordial times - categories - have ceased to signify 'the transcendental ideality of appearances,' resulting in lapsing into 'numerical formulas' (B206) - the 'transcendental principle of the mathematics of appearances' (B206). We think that the conundrums in regard to mathematics and Kant's metaphysics originate in this alienation of numbers from 'the transcendental ideality of appearances' while this alienation enabled humans to metamorphose 'natural numbers in primordial times' - categories - into 'natural or real numbers' in mathematics, enhancing them to 'synthetic a priori principles' (B305)" (YAMAMOTO 2017d: 19-29). Who has played a major role in enhancing "natural numbers in primordial times" to "synthetic a priori principles"? We think that it is Leonhard Euler (1707-1783), who discovered "the zeta-function" (TANTON 2005: 535-536), and consequently " $e^{i \pi}+1=0$ " (TANTON 2005: 175-176). Second, when Kant, who firmly believed that "the propositions of geometry are cognized synthetically a priori and with apodictic certainty," met "the antinomy of pure reason," he was forced to take refuge in "transcendental dialectic" (B88), inventing "a thing in itself" which, he believes, "remains entirely unknown to us" (A42) or "is entirely beyond our cognitive sphere" (A190). We have already made a discourse in regard to this issue, saying, "Kant's difficulty in his metaphysics is related to the issue of 'the absolute totality of series of conditions in the world of sense,' in connection with the 'issue about the absolute magnitude of the series in this world' (A515/B543-A516/B544), which looms as 'the antinomy of pure reason in its cosmological ideas' (A506/B534)...From our viewpoint, Kant's discourse seems to testify that Kant has made a mistake, which forced him to fabricate a thing in itself, which is supposedly an epistemological naught" (YAMAMOTO 2017a: 19-37). Third, Kant's revolutionary way of thinking in the Critique of Pure Reason, which tries to solve the problems in regard to "all propositions of geometry" by means of inventing "a thing in itself" has not only failed to attain its aim but has created another problem; the belief that "an unknown something" (B312) "remains entirely unknown to us" or "is entirely beyond our cognitive sphere." While mathematicians need to know "an unknown something" in order to make mathematics stand on a solid foundation, the way to reach it is closed by Kant, who asserts that it "remains entirely unknown to us" or "is entirely beyond our cognitive sphere."

Frege, who thinks that "we shall do well in general not to overestimate the extent to which arithmetic is akin to geometry" (FREGE 1980: 1-24), refers to Leibniz's warning, which says, "one geometrical point, considered by itself, cannot be distinguished in any way from any other; the same applies to lines and planes" (FREGE 1980: 1-24). In other words, "the points or lines or planes which we intuit are not really particular at all, which is what enables them to stand as representatives of the whole of their kind" (FREGE 1980: 1-24). Therefore, when Frege suggests that the "axioms of geometry are independent of one another and of the primitive laws of logic, and consequently are synthetic" (FREGE 1980: 1-24), we entirely agree with him, thinking that "axioms of geometry" cannot belong to "synthetic a priori propositions," but belong to a "synthetic proposition." On the contrary, "the truths of arithmetic govern all that is numerable. This is the widest domain of all; for to it belongs not only the actual, not only the intuitable, but everything thinkable" (FREGE 1980: 1-24). Therefore, we 
have to think that the "truths of arithmetic" do not parallel "axioms of geometry," but precede it. Nevertheless, when we hear from Frege, who says, "if the general inclination is, on the whole, to hold that Number is indefinable, that is more because attempts to define it have failed than because anything has been discovered in the nature of the case to show that it must be so" (FREGE 1980: 2439), we are perplexed and annoyed. At the same time, Frege suggests which direction we should go, saying, "no one has ever been able to give a definition of the property 'one.' LEIBNIZ indeed says that 'By one is meant whatever we grasp in one act of the understanding,' but this is to define 'one' in terms of itself. Besides, surely we can also grasp what is many in one act of the understanding?" (FREGE 1980: 39-67). Yes, we agree with what Frege refers to in regard to the property of "one," thinking that 1) "one" would be the most primordial number among humans, 2) Leibniz's discourse in regard to "one" is quite brilliant. Indeed, we have to think that number " 1 " corresponds to "one act of the understanding." Here, we should like to add, saying, that when "one act of the understanding" is to be carried out by the representation I think, it signifies "the position of each existence" (B262), determining "the representation I think." In other words, one act of the understanding or many acts of the understanding or myriads of acts of the understanding could be correspondent to the representation I think - "oneness" - "an infinite set of representations within itself" (B40). "This is to define "one' in terms of itself." Since the "act of the understanding" - "one," i.e., number "1" - is the "necessity" (B111) which "is nothing other than the existence" (B111), namely the representation I think itself, we can say that 1) one act of the understanding or many acts of the understanding or myriads of acts of the understanding - number " 1 " - is commensurate with the "possibility itself," 2) number "1" - the representation I think - belongs to "synthetic a priori propositions" (B205), i.e., "axioms" (B205). Thus, "oneness," i.e., number "1" arises in virtue of "a priori principles of the possibility of experience." In regard to this issue, we have already said that "1) 'one consciousness,' or 'one experience,' or 'one reality' is homogeneous with 'one representation' (A99), 2) since 'the unity of the synthesis of the manifold of a homogeneous intuition in general' (A143) arises as a result of cognizing space-time itself - death itself - in 'the apprehension of the intuition' as 'a representation,' 'one representation' is commensurate with 'the representation I think' (B132) - a human or humans - which is supposed to be 'a new bridge between the countable assemblage and the continuum' (HILBERT 1902: 437-479), namely $i^{2}=-1$, 3) this representation 'summarizes the successive addition of one (homogeneous) unit to another,' thereby producing the natural numbers in mathematics, which 'are thought in accordance with the unity of the categories' (B305-A249)" (YAMAMOTO 2018: 20-45). In addition, we would like to stress that 1) the natural number in the most primordial time would be number "1," 2) number "1" would be, from the beginning, the bridge between quantum continuum and "quantum discretum" (A526/B554) - "the appearance as quantum continuum" (A527/B555) and the appearance as "the multiplicity of units" (A527/B555) - which have the properties of "the infinite divisibility" and "decomposition to infinity," 3 ) therefore, number " 1 " -

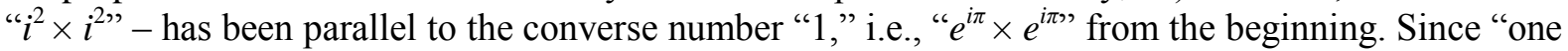
consciousness," or "one experience," or "one reality," which is homogeneous with "one representation" (A99) - the cardinality in the domain; " $0 \rightarrow 1 \rightarrow i^{2}=-1 \rightarrow 0$ " or " $0 \rightarrow i^{2}=-1 \rightarrow 0$ " is commensurate, from the beginning, with "the unity of the synthesis of the manifold of a homogeneous intuition in general" (A143), a human - number "1" - is homogeneous, from the beginning, with "a kind of metaphysical figure" (FREGE 1980: 24-39) or "symbols for the syllogistic figures” (FREGE 1980: 24-39).

Therefore, when Frege says, "It would indeed be remarkable if a property abstracted from external things could be transferred without any change of sense to events, to ideas and to concepts" (FREGE 1980: 24-39), we agree with him, thinking that first-order logic signifies a human or number " 1 " or a representation with "a property abstracted from external things" - a "metaphysical figure" itself. Therefore, a metaphysical figure itself can transfer "a property abstracted from external things" "abstracted relations" (B57) - "without any change of sense to events, to ideas and to concepts," e.g., to number " 1 " or to prime number. In regard to "number," we have already made a discourse, saying, "When the 'pure schema of magnitude (quantitatis), ' i.e., ' 0 ' or ' 1 ,' is assigned to 'a representation' for 'the successive addition of one (homogeneous) unit to another' (B182), 'the representation I think' performs it. Since 'one (homogeneous) unit' signifies 'allness (totality)' or 'a unity' or 'a whole' (A524/B552), which is correspondent to 'the pure image of all magnitudes (quantorum),' which can 
be thought to signify either 'amount' (B215) of magnitude in filled space-elapsing time - the intensive magnitude - or 'volume' (B215) of magnitude in nullity in space-time - the extensive magnitude - we have to say that 'a representation' is counting 'how-many-times' (B300) a part of 'the intensive magnitude' has disappeared in the domain, which corresponds to how-many-times 'the extensive magnitude' has appeared in the converse domain when it is summarizing the successive addition of one (homogeneous) unit to another" (YAMAMOTO 2018: 20-45). Furthermore, our metaphysical exploration - an attempt to rectify the foundation of Kant's "transcendental philosophy" (B29) - led us to the findings that 1) a number, which "belongs to the category of allness" (B111) signifies the unity of the categories, 2) the number pertains to "self-thought a priori first principles of our cognition" (B167) drawn from possible experience, and to the "subjective predispositions for thinking, implanted in us along with our existence (B167), 3) "the category of allness" and "the unity of the categories" - "oneness," i.e., "an infinite set of representations within itself" - is nothing but the product of synthesis speciosa and synthesis intellectualis, 4) synthesis speciosa would operate in a reciprocal and converse parallel to synthesis intellectualis, enabling us to yield "the possibility of the category of community" (B292) and the possibility itself of "the category of community" - "quantum discretum" or "quantum continuum." Therefore, "quantum continuum" or "quantum discretum" "pure schema of magnitude (quantitatis)" - is to be "subject to the law of everything alterable" (A540/B568) in its space-time-determination "that everything that happens must find its cause in the appearances (of the previous state)" (A540/B568) (YAMAMOTO 2017b: 72-81, YAMAMOTO 2017c: 57-70, YAMAMOTO 2017d: 19-29, YAMAMOTO 2018: 20-45). When Frege says, "How is it after all that we do become acquainted with, let us say, the Number of figures of the syllogism as drawn up by Aristotle? ... What we see is at most certain symbols for the syllogistic figures, not the figures themselves. How are we to be able to see their Number, if they themselves remain invisible?" (FREGE 1980: 24-39), we say that it is the "transcendental analytic" that enables us to see "the Number of figures of the syllogism" (YAMAMOTO 2016: 87-100, YAMAMOTO 2017a: 19-37, YAMAMOTO 2017b: 72-81, YAMAMOTO 2017c: 57-70, YAMAMOTO 2017d: 19-29, YAMAMOTO 2018: 20-45). We should like to say, in an opposite manner to what Frege says (FREGE 1980: 24-39), that "Number is definable, that is because attempts to define it have succeeded and because the law has been discovered in the nature of the case to show that it must be so." It is obvious that in order for the "Number" to be definable as "the syllogistic figures" themselves, it is absolutely necessary that "the Number of figures of the syllogism" or a metaphysical figure itself could be grounded on "universal cognitions a priori" (A300) - nullity in space-time (i.e., a priori intuition or the infinite divisibility) as death itself (i.e., pure intuition or decomposition to infinity) which enables it to "serve as the major premise in a syllogism" (A300). Yes, they can, because the Number of figures of the syllogism or a metaphysical figure itself has arisen as first-order logic, i.e., the converse "general logic." From our discourse so far (YAMAMOTO 2016: 87-100, YAMAMOTO 2017a: 19-37, YAMAMOTO 2017b: 72-81, YAMAMOTO 2017c: 57-70, YAMAMOTO 2017d: 1929, YAMAMOTO 2018: 20-45), it is obvious that the Number of figures of the syllogism or a metaphysical figure itself is tantamount to "the representation I think" (B132) or "the cognition itself a priori in intuition" which is equivalent to a human or Homo sapiens. Here, an apparently insolvable problem could loom, which would be the last obstacle to reach a denouement. Frege succinctly refers to it as follows; "Why do we call things units, if 'unit' is only another name for thing, if any and every thing is a unit or can be regarded as one? E. SCHRÖDER gives as the reason, that the word is used for ascribing to the items that are to be numbered the necessary identity" (FREGE 1980: 39-67). How do we give "unit" - "the items that are to be numbered" - identity? "If we provide the I with differentiating strokes, it becomes unusable for arithmetic" (FREGE 1980: 39-67). Therefore, we absolutely need the "necessity," namely "the existence that is given by possibility itself" - "first-order logic" - in order to "provide the I with differentiating strokes," namely "the necessary identity." What does the "necessity," i.e., first-order logic mean? Obviously, it means a human or Homo sapiens, which could be homogeneous with the category and the categories, i.e., "number." If someone asks; how could first-order logic, which is correspondent to the Number of figures of the syllogism or a metaphysical figure itself, manifest? We answer, saying that the Number of figures of the syllogism or a metaphysical figure itself could manifest as "one and itself," which is to signify "one thing (numerica identitas)" (B319). This is first-order logic - one representation, i.e., the cardinality in the domain; " $0 \rightarrow 1 \rightarrow i^{2}=-1 \rightarrow 0$ " or " $0 \rightarrow i^{2}=-1 \rightarrow 0$ " - which signifies "noting other than principles of the determination of the existence of appearances" (B262) in space-time, in accordance with all 
three of its modi: "a magnitude (the magnitude of existence, i.e., duration)" (B262), "a series (one after another)" (B262) and "a sum of all existence (simultaneous)" (B262). We should like to stress in regard to "the existence that is given by possibility itself" that, all three of the modi of first-order logic, which consists in "pure a priori concepts" (A95) such as "a magnitude," "a series" and "a sum," indicates that first-order logic signifies "a modus of the existence of that which lasts and persist" (B227) - "the object itself, i.e., the substance (phaenomenon)" (B227).

\section{ONENESS, Numbers, AND CAUSALITY}

From the discourse above, it follows that we can think that 1) "oneness," i.e., "an infinite set of representations within itself" signifies "allness (totality)" (B111), i.e., the category of allness and "a whole," i.e., the unity of the categories, 2) allness (totality), i.e., the category of allness - "number ( $\mathbb{N}$ $=\{1,2,3,4, \ldots\}$ and $\left.i^{2}=-1\right) "$ - and a whole, i.e., the unity of the categories - prime number - signify "the possibility of the category of community" (B292) or the possibility itself of the category of community, 3$)$ "number $\left(\mathbb{N}=\{1,2,3,4, \ldots\}\right.$ and $\left.i^{2}=-1\right)$ " and prime number stand under a third thing, i.e., $\left\{i^{2} \cdot e^{i \pi}\right\}=$ "1," indicating that allness (totality), e.g., "number $\left(\mathbb{N}=\{1,2,3,4, \ldots\}\right.$ and $\left.i^{2}=-1\right)$ " could be commensurate with the unity of the categories, e.g., prime number. Therefore, we are annoyed when we hear from Frege that "the difficulty is so well hidden under the word 'unit,' that those who have any suspicion of its existence must surely be few at most" (FREGE 1980: 39-67). We think that the Frege's remark arises from his belief that "if we try to produce the number by putting together different distinct objects, the result is an agglomeration in which the objects contained remain still in possession of precisely those properties which serve to distinguish them from one another; and that is not the number" (FREGE 1980: 39-67). Contrary to what Frege refers to, we say, "if we try to produce the number ' 1 ' by putting together different distinct objects; a filled space-elapsing time and empty space-nullified time, the result is "oneness" in which the objects contained remain still in possession of precisely those properties which serve to distinguish them from one another; and that is the number ' 1 '." We would like to add, saying, in an opposite manner to what Frege refers to (FREGE 1980: 39-67), that "this is outward manifestation of an inward process of thought, and no illusion of one...word full of thought possesses some mysterious power, since what is different is to be made identical simply by being called a unit or the category." When Frege asks, saying, "Why do we ascribe identity to objects that are to be numbered? And is it only ascribed to them, or are they really identical? In any case, no two objects are ever completely identical" (FREGE 1980: 39-67), we would say, "we are allowed to ascribe identity to 'objects in themselves in which the absolutely unconditioned might possibly occur' (A508/B536) that are to be numbered, since in any case, objects in themselves are ever completely identical." How do we pertain to "objects in themselves, which manifest as the possibility itself of possible experience or the possibility of experience itself" in the world of sense? We pertain to "objects in themselves" through the "intensive magnitude, i.e., a degree of influence on sense" (B208) in which the absolutely unconditioned might possibly occur. Since the "intensive magnitude, i.e., a degree of influence on sense" corresponds to the "degree of reality" (B210), i.e., "the moment of gravity," if the degrees are lost simultaneously, "a sum total of mere relations" (B321) and "the inner determinations of a substantia phaenomenon" (B321) - the moment of gravity - could become commensurate with "the pure image of all magnitudes (quantorum)" (B182) - "numerical magnitudes" (B221), namely the converse moment of gravity. Furthermore, since "the pure image of all magnitudes (quantorum)" is commensurate with "number," i.e., "pure schema of magnitude (quantitatis)" (B182), we think that 1) the pure image of all magnitudes (quantorum) - "the image itself" (A140) - signifies the converse domain while "pure schema of magnitude (quantitatis)," namely number $\left(\mathbb{N}=\{1,2,3,4, \ldots\}\right.$ and $\left.i^{2}=-1\right)$ signifies "the image itself" and the "light" itself. Since pure schema of magnitude "abstracts from all content of cognition" (B102), "number" - the image itself and the light itself - seems to be commensurate with "general logic" itself, which "expects that representations will be given to it from elsewhere" (B102). Representations must be given to general logic, from the world of sense, i.e., the domain, through the disappearance of "first-order logic," which is to occur necessarily and universally in the domain. Therefore, we say that first-order logic could be affiliated with the converse domain. However, it is clear that general logic is distinct from first-order logic on account of the fact that general logic - the converse domain - is "a pure category, in which abstraction is made from any condition of sensible intuition" while first-order logic, comprising "all content of cognition" (B102), is determined to abstract from "all content of cognition." We dare to say that general logic, which consists in "the field 
or the entire object of possible experience" (A95), could signify quantum continuum, while first-order logic which consists in the field or the entire "objects of experience" (B258), could signify quantum discretum - "objects of sensibility" (B320). Furthermore, quantum continuum, i.e., the elements of "the entire object of possible experience" - the extensive magnitude, i.e., "the manifold of pure intuition" (B104-A79) - has a one-one relationship, in a manner of the "instantaneous and simultaneous reciprocity-converse," with quantum discretum, i.e., "objects of sensibility." Thus the "objects of sensibility" - the "dynamical categories" - are commensurate with "the manifold of sensible representation (intuition)" which is "represented and apprehended by us as extensive" (204) the converse moment of gravity - or "the manifold of given representations" (B143) which is "already intuited as aggregates (multitudes of antecedently given parts)" (B204) - the moment of gravity.

From the discourse so far, it follows that we can think that the domain, e.g., the "aggregates (multitude of antecedently given parts)" - the "degree of reality" which may be called "as cause a 'moment,' e.g., the moment of gravity" (B210) or the intensive magnitude - could be parallel to the converse domain, e.g., the extensive magnitude - "that magnitude the apprehension of which is not successive but instantaneous" (A169), thereby being continuous. Furthermore, we have already found that "the manifold of given representations" (B143) and "the manifold of pure intuition," i.e., "the manifold of sensible representation (intuition)" (A129) - the domain and the converse domain signify "the system of all cardinal numbers or of all Cantor's alephs" (HILBERT 1902: 437-479) the cardinality in the domain; " $0 \rightarrow 1 \rightarrow i^{2}=-1 \rightarrow 0$ " or " $0 \rightarrow i^{2}=-1 \rightarrow 0$ " and the cardinality in the converse domain; " $0 \rightarrow 1 \rightarrow e^{i \pi}=-1 \rightarrow 0$ " or " $0 \rightarrow e^{i \pi}=-1 \rightarrow 0$ " (YAMAMOTO 2018: 20-45). This system, which signifies the cause and causality (YAMAMOTO 2017c: 57-70, YAMAMOTO 2017d: 19-29), is grounded in "dynamical categories," i.e., "metaphysical categories" (YAMAMOTO 2017d: 19-29). Since the cardinality in the domain apparently pertain to " 0 ," the schemata indicates that when "the manifold of given representations" - "inductive numbers" - necessarily and universally goes through " 0, ," they would metamorphose to the "reciprocal-converse manifold of given representations" - natural number $(\mathbb{N}=\{1,2,3,4, \ldots\})$. Seeing that all elements of $e^{i \pi}$ are derived from a natural number $(\mathbb{N}=\{1,2,3,4, \ldots\})$ and the cardinality in the domain (" $0 \rightarrow 1 \rightarrow i^{2}=-1 \rightarrow 0$ " or " 0 $\rightarrow i^{2}=-1 \rightarrow 0$ "), we can say that that $e^{i \pi}$ is composed of natural number $(\mathbb{N}=\{1,2,3,4, \ldots\})$ and " $i^{2}=$ -1 ." Therefore, the domain, which consists in "inductive numbers" and the cardinality, i.e., " $0 \rightarrow 1 \rightarrow$ $i^{2}=-1 \rightarrow 0$ " or " $0 \rightarrow i^{2}=-1 \rightarrow 0$ " "constitute the field or the entire object of possible experience" (A95) on account of the fact that it is combined with the converse domain, composed of natural number $(\mathbb{N}=\{1,2,3,4, \ldots\})$ and converse " $i{ }^{2}=-1$," namely " $e e^{i \pi}=-1$." In regard to the issue of the domain and the converse domain, and their relation to "the Platonic mathematical world" (PENROSE 2007: 7-24, 1010-1047), we have already said, "We think that if the Platonic mathematical world manifests as 'the transcendental ideality of appearances' (A506/B534) or 'synthetic a priori principles' (B305), i.e., 'transcendental truth,' it would last forever irrespective of the existence of humans on this planet...Since 'nowhere beyond the field of possible experience can there be any synthetic a priori principles' (A248-B305), 'all of our cognitions' (A146), which 'lie in the entirety of all possible experience' (A146), can be enhanced to 'synthetic a priori principles' (B305), i.e., 'transcendental truth, which precedes all empirical truth and makes it possible' (A146). Since all of our cognitions, which can be enhanced to 'synthetic a priori principles,' belong among categories, the category, or categories are the crucial elements through which the Platonic mathematical world would manifest as the converse domain with regard to the physical world, i.e., the domain. If 'there is a oneone relation whose domain is the one class and whose converse domain is the other' (RUSSELL 1971: 52-62), in other words, if there is a one-one relation between the Platonic mathematical world and the physical world, we could know what kind of class the Platonic mathematical world is endowed with, since we have already clarified that the class in the physical world is the cardinality as ' $0 \rightarrow 1 \rightarrow i^{2}=$ $1 \rightarrow 0$ ' or ' $0 \rightarrow i^{2}=-1 \rightarrow 0$ '” (YAMAMOTO 2018: 20-45).

Since the cardinality in the domain and that in the converse domain seem to have a one-one relation (YAMAMOTO 2018: 20-45), the cardinality in the converse domain; " $0 \rightarrow 1 \rightarrow e^{i \pi}=-1 \rightarrow 0$ " or " 0 $\rightarrow e^{i \pi}=-1 \rightarrow 0$ " can also belong to "synthetic a priori propositions." Once the cardinality in the domain and that in the converse domain have been found to have a one-one relation, it is absolutely necessary to show how the domain is linked with the converse domain. If it is not shown, what Kant refers to could happen: "it is entirely contradictory and impossible that a concept should be generated completely a priori and be related to an object although it neither belongs itself within the concept of 
possible experience nor consists of elements of a possible experience" (A95). It is absolutely necessary to show that "the concept of possible experience" (A95), namely "an a priori concept" (A95) and the "elements of a possible experience" (A95) are necessarily and universally (in other words, synthetically a priori) combined, thereby yielding "pure a priori concepts" (A95) - "the concept itself" (A95). If it is attained, we can say that 1) "pure a priori concepts" rest on "synthetic $a$ priori cognitions of things" (A247), 2) "pure a priori cognition" (A87) in regard to "pure a priori concepts" is homogeneous with "the cognition itself a priori in intuition," namely the mental world, 3) "the field or the entire object of possible experience" (A95), which signifies the physical world and the mental world, corresponds to "the field of its pure a priori cognition" (A73) or "the field of possible experience" (A248-B305), which signifies the Platonic mathematical world and the mental world, 4) "pure a priori cognition" is to be correspondent to "the categorical syllogisms" (A406), which can make "the unconditioned unity of the subjective conditions of all representations" (A406) and "the unconditioned unity of objective conditions in appearances" (B433) its content. We have a device with which the domain and the converse domain are combined. What is it? It is relationnumbers; "a median class having $\aleph_{0}$ terms, i.e., $e^{i \pi}=-1=i$ " (YAMAMOTO 2018: 20-45) and $\left\{i^{2} \cdot e^{i \pi}\right\}$. Here, we acknowledge that the relation-numbers obeys "the usual formal laws of addition, multiplication, and exponentiation" (RUSSELL 1971: 89-96). Accordingly, the commutative law: $\alpha+$ $\beta=\beta+\alpha$ and $\alpha \times \beta=\beta \times \alpha$, the associative law: $(\alpha+\beta)+\gamma=\alpha+(\beta+\gamma)$ and $(\alpha \times \beta) \times \gamma=\alpha \times(\beta \times \gamma)$, the distributive law: $\alpha(\beta+\gamma)=\alpha \beta+\alpha \gamma$, and the laws of exponentiation: $\alpha^{\beta} \cdot \alpha^{\gamma}=\alpha^{\beta+\gamma}, \alpha^{\gamma} \cdot \beta^{\gamma}=(\alpha \beta)^{\gamma}$, $\left(\alpha^{\beta}\right)^{\gamma}=\alpha^{\beta \gamma}$ all hold for "a median class having $\aleph_{0}$ terms, i.e., $e^{i \pi}=-1=i^{2}$."

At this stage of the discourse, we have to discuss the issue of the "multiplicative axiom" (RUSSELL 1971: 117-130). According to Russell, the problem of multiplication arises as follows: "Suppose we have a class $k$ consisting of classes; suppose the number of terms in each of these classes is given. How shall we define the product of all these numbers?" (RUSSELL 1971: 117-130). When Russell says, "It is the case when $k$ is infinite, even though its members may be finite, that we have to find a way of dealing with," we think that it is possible to find a clue to the solution - "prime number." When number " 1 " or "inductive prime number" in the domain necessarily and universally goes through " 0 ," it is metamorphosed into the converse number " 1 " or the "converse prime number." The "synthesis of representations" (A155) (thereby pure synthesis of representations), e.g., the synthesis of number " 1 " with the converse number "1" by means of "a median class having $\aleph_{0}$ terms, i.e., $e^{i \pi}=-1$ $=i^{2}$ " yields "oneness." Likewise, the synthesis of inductive prime number with converse prime number yields prime number which has the property of a real number. Therefore, we say that 1) number "1" or prime number in the domain is composed of the same element as that in the converse domain is composed of, 2) since number " 1 " or prime number in the domain corresponds to a filled space-elapsing time while that in the converse domain corresponds to nullity in space-time, number "1" or prime number can be thought to have the same element as nullity in space-time - space-time itself - indicating that number " 1 " or prime number in the domain is, in a manner of "instantaneous and simultaneous reciprocity-converse," equivalent to "number $\left(\mathbb{N}=\{1,2,3,4, \ldots\}\right.$ and $\left.i^{2}=-1\right)$," i.e., "a composite (compositum reale)" (B262), thereby to "real number $\left(i^{2}=-1\right.$ and $\left.\left.\mathbb{R}=\{1,2,3,4, \ldots\}\right), " 3\right)$ thus number " 1 " or prime number, which stands under "the usual formal laws of addition, multiplication, and exponentiation," could belong to the "synthetic a priori propositions," which are to be correspondent to "synthetic a priori judgments" or "synthetic a priori principles." This is how to "define 'one' in terms of itself" (FREGE 1980: 39-67). We, in agreement with Leibniz, have already made the discourse above, saying that "one act of the understanding or many acts of the understanding or myriads of acts of the understanding could be correspondent to the representation I think" - "one and itself." We can think that one act of thinking of death itself as the "possibility itself" of "possible experience" - the reciprocity-converse in a instantaneous and simultaneous event of thinking - is commensurate with "one and itself," which indicates that "the pure intuitions as well as the pure concepts of the understanding" (B166) are the "elements of cognition that are to be encountered in us a priori." This is drawn from the metaphysical deduction and the transcendental deduction, in which "objects of an intuition" (B159) are cognized a priori in virtue of "objects of possible experience," indicating that "no a priori cognition is possible for us except solely of objects of possible experience" (B166). Therefore, when Kant says, "Now there are only two ways in which a necessary agreement of experience with the concepts of its objects can be thought: either the experience makes these concepts possible or these concepts make the experience possible" (B166-B167), we have to put 
it another way, saying that "there are only two ways in which a priori cognition can be thought: either possible experience makes objects of possible experience possible, or objects of possible experience make possible experience possible." "A necessary agreement of possible experience with objects of possible experience" - a priori cognition - stands under the condition that "with regard to the pure intuitions as well as the pure concepts of the understanding, there are elements of cognition that are to be encountered in us a priori" (B166) - "the idea" (A489/B517) which "encounters its object within the world" (A489/B517). What is the idea which "encounters its object within the world" and "goes beyond the possibility of experience" (B377)? It is "empirical intuition" whose object is a priori intuitions - a pure category - which are to be commensurate with "objects of possible experience," namely "the field of possible experience." Therefore, "the conditions of the unity of empirical cognition in the synthesis of the appearances" (A181-B224) - the conditions of the moment of gravity - stand under "the field of possible experience," or "the field of possible empirical cognition" (A644/B672) - "the field of decomposition to infinity." We dare to say that 1) the "empirical cognition in the synthesis of the appearances" - the moment of gravity (thereby the infinite divisibility) - is possible through "a priori cognition in regard to decomposition to infinity," 2) the moment of gravity (thereby the infinite divisibility) - "every empirical representation of the manifold" (A139) - is to be commensurate with a manifold of sensibility, 3) a priori cognition in regard to the death itself is possible through a priori cognition in regard to the disappearance of "the things themselves, which appear." Since a priori cognition in regard to the death itself is commensurate with the synthetic a priori judgments in regard to "one thing (numerica identitas)" - the moment of gravity or the light itself - it signifies "one experience" (A108), or "one concept" (A142) or "one cognition" (A108) or "one moment" (A99) or "one representation" (A99) or "one subject" (A265), or "one totality" (A155), or "one complete whole" (A676/B704). The "oneness," which rests on "synthetic $a$ priori judgments," is nothing but "synthetic a priori cognition," indicating that the possibility of "oneness" corresponds to the "possibility itself" of "one." Since "one totality," or "one complete whole" is correspondent to the "possibility itself" of "one," we have to say that the unconditioned such as "one totality," or "one complete whole" signifies "oneness itself" - "one and itself." "The unconditioned," which is conditioned by "oneness" - oneness itself, i.e., an appearance - "belongs to the category of allness" (B111), i.e., "Totality" (B106), which signifies the "unity of the categories" (A249), i.e., "Unity" (B106). "Totality" or "Unity" are nothing but "pure a priori concepts" "number $\left(\mathbb{N}=\{1,2,3,4, \ldots\}\right.$ and $\left.i^{2}=-1\right)$ " - while the unconditioned, which is conditioned by oneness is homogeneous with a priori categories or a synthetic a priori concept - a human or Homo sapiens. Thus, a synthetic a priori concept makes pure a priori concepts possible. When Kant says, "whence will one derive the character of the possibility of an object that is thought by means of a synthetic $a$ priori concept, if not from the synthesis that constitutes the form of the empirical cognition of objects?" (A220), we, thinking that "the character of the possibility of an object" corresponds to a human or Homo sapiens, say that it is humans' a priori cognition in regard to "a synthetic a priori concept," such as the moment of gravity or death itself or the light itself, that leads them to a priori cognition in regard to the category and the categories, namely the category of allness, e.g., "number $\left(\mathbb{N}=\{1,2,3,4, \ldots\}\right.$ and $\left.i^{2}=-1\right)$ " and "the unity of the categories," e.g., prime number. "The category of allness," as "the unity of the categories," is deemed to be equivalent to "one and itself," namely "oneness itself," which is commensurate with "A thing $=A$, which is something = $B$ " (B192). Therefore, we dare to say that the "Unity of Quantity," "Plurality of Quantity" and "Totality of Quantity" in "Table of Categories" (B106) all pertain to "one and itself" - "an infinite set of representations within itself."

In view of the fact that oneness itself - number " 1 " or prime number or one and itself - all pertain to "elements of cognition that are to be encountered in us a priori," we conclude that they belong to "universal propositions a priori" in virtue of synthetic a priori propositions. Therefore, when Kant makes a discourse in regard to "the relation of community or interaction" (B258) among "objects of experience" (B258) that "the simultaneity of substances in space cannot be cognized in experience otherwise than under the presupposition of an interaction among them; this is therefore also the condition of the possibility of the things themselves as objects of experience" (B258), we have to say, in an opposite manner, that "though the simultaneity of substances in space-time cannot be cognized in experience itself under an interaction among them, it can be cognized in 'perceptions as one that is present in the subject when the other is not' (B257) - 'the synthesis of the imagination in 
apprehension' (B257) - which stands under 'the categorical syllogisms, whose major premise, as a principle, states' the relation of 'contradictorily opposed predicates in one and the same object' to 'contradictorily opposed determinations in the existence of one and the same thing; a priori cognition in regard to the infinite divisibility or decomposition to infinity of 'the things themselves, which appear" - "Planck's constant" (PENROSE 2007: 493-526) - is therefore also the condition of the possibility itself of the things themselves as objects of possible experience."

Here, we recollect the cardinality in the domain; " $0 \rightarrow 1 \rightarrow i^{2}=-1 \rightarrow 0$ " or " $0 \rightarrow i^{2}=-1 \rightarrow 0$," which belongs to "synthetic a priori propositions" - "axioms." The cardinality in the domain can be thought to signify first-order logic, while number "1" is to signify a metaphysical figure itself. From the discourse above, we would rewrite the cardinality as " $0 \rightarrow$ ' 1 ' or prime number or one and itself $\rightarrow i^{2}$ $=-1 \rightarrow 0$ " or " $0 \rightarrow i^{2}=-1 \rightarrow 0$." It is obvious that " 1 " corresponds to $i^{2} \times i^{2}=-1 \times-1=1$, which corresponds to " $\left\{i^{2} \cdot i^{2}\right\} \cdot\left\{i^{2} \cdot i^{2}\right\} \cdot\left\{i^{2} \cdot i^{2}\right\} \cdot\left\{i^{2} \cdot i^{2}\right\} \cdots \infty$." Therefore, it becomes possible to rewrite the cardinality in the domain as follows: " $0 \rightarrow$ ' 1 ' or prime number or one and itself, namely $\left|\left\{i^{2} \cdot i^{2}\right\} \cdot\left\{i^{2} \cdot i^{2}\right\} \cdot\left\{i^{2} \cdot i^{2}\right\} \cdot\left\{i^{2} \cdot i^{2}\right\} \cdots\right| \rightarrow\left|\left\{i^{2} \cdot i^{2}\right\} \cdot\left\{i^{2} \cdot i^{2}\right\} \cdot\left\{i^{2} \cdot i^{2}\right\} \cdot\left\{i^{2} \cdot i^{2}\right\} \cdots\right| \times i^{2}=-1 \rightarrow 0$." Since there is "a median class having $\aleph_{0}$ terms, i.e., $e^{i \pi}=-1=i^{2}$ " between the domain and the converse domain, we rewrite it again as follows: " $0 \rightarrow\left|\left\{i^{2} \cdot e^{i \pi}\right\} \cdot\left\{i^{2} \cdot e^{i \pi}\right\} \cdot\left\{i^{2} \cdot e^{i \pi}\right\} \cdot\left\{i^{2} \cdot e^{i \pi}\right\} \cdots\right| \rightarrow$ $\left|\left\{i^{2} \cdot e^{i \pi}\right\} \cdot\left\{i^{2} \cdot e^{i \pi}\right\} \cdot\left\{i^{2} \cdot e^{i \pi}\right\} \cdot\left\{i^{2} \cdot e^{i \pi}\right\} \cdots\right| \times i^{2}=-1$ or $e^{i \pi}=-1 \rightarrow 0$." The $\left\{i^{2} \cdot e^{i \pi}\right\}$ indicates that the multiplication of number " 1 " or prime number in the domain by $i^{2}=-1$ takes place simultaneously with the multiplication of number " 1 " or prime number in the converse domain by $e^{i \pi}=-1$. Therefore, it can be said that 1) number " 1 " or prime number in the domain and that in the converse domain have a one-one relation, 2) the "one-one relation" is meant to be the "instantaneous and simultaneous reciprocity-converse" - "abstracted relations" (B57) - "that cannot occur without the restrictions that nature has attached to them" (B57), 3) therefore the instantaneous and simultaneous reciprocityconverse, is the bridge between the domain and the converse domain - "the schematism of the understanding through the transcendental synthesis of imagination" (B185). The "abstracted relations" are commensurate with the property of number " 1 " or prime number or one and itself, which comprises "the function that corresponds to inner sense (to a receptivity)" (B185). We think that the instantaneous and simultaneous reciprocity-converse - "abstracted relations" - is parallel to "LEIBNIZ's definition" of identity, which says, "Things are the same as each other, of which one can be substituted for the other without loss of truth" (FREGE 1980: 67-99). Since number "1" or prime number or one and itself has the property of "relation-numbers" - "a median class having $\aleph_{0}$ terms, i.e., $e^{i \pi}=-1=i^{2}$ and $\left\{i^{2} \cdot e^{i \pi}\right\}=1$ " - it becomes possible to say that 1 ) number "1" or prime number or one and itself can serve as "true correlate, i.e., the thing in itself" (A30) - "wavefunctions" (PENROSE 2007: 493-526) - for space-time-determination either in the domain, e.g., "impenetrability in matter, as empirical intuition, does" (B278), or in the converse domain, e.g., "intellectual representation of the self-activity of a thinking subject" (B278) which "is determinable through the manifold of those appearances" $(\mathrm{A} 251), 2)$ the number "1" or prime number or one and itself $-\left\{i^{2} \cdot e^{i \pi}\right\}=1-$ is to stand under the "formal laws of addition, multiplication, and exponentiation." In addition, we say that number " 1 " or prime number or one and itself - "the synthetic unity of the manifold of intuition in a possible experience" (A158), e.g., "number ( $\mathbb{N}=\{1$, $2,3,4, \ldots\}$ and $\left.i^{2}=-1\right)$ " - is "the necessary conditions of the synthetic unity" (e.g., $\left\{i^{2} \cdot e^{i \pi}\right\}=1$ and $e^{i \pi}=$ $-1=i^{2}$ ), under which "every object stands" (A158). Thus, the number "1" or prime number or one and itself - a metaphysical figure itself, i.e., the major premise of categorical syllogism - is homogeneous with first-order logic, namely "the dynamical law of causality" (A228). We think that first-order logic is commensurate with the cardinality in the domain; " $0 \rightarrow 1 \rightarrow i^{2}=-1 \rightarrow 0$ " or " $0 \rightarrow i^{2}=-1 \rightarrow 0$," which can be rewritten as " $0 \rightarrow$ ' 1 ' or prime number or one and itself $\rightarrow i^{2}=-1 \rightarrow 0$ " or " $0 \rightarrow i^{2}=-1$ $\rightarrow 0$," which is equivalent to " $0 \rightarrow$ ' 1 ' or prime number or one and itself, namely $\left|\left\{i^{2} \cdot i^{2}\right\} \cdot\left\{i^{2} \cdot i^{2}\right\} \cdot\left\{i^{2} \cdot i^{2}\right\} \cdot\left\{i^{2} \cdot i^{2}\right\} \cdots\right| \rightarrow\left|\left\{i^{2} \cdot i^{2}\right\} \cdot\left\{i^{2} \cdot i^{2}\right\} \cdot\left\{i^{2} \cdot i^{2}\right\} \cdot\left\{i^{2} \cdot i^{2}\right\} \cdots\right| \times i^{2}=-1 \rightarrow 0$," which is equivalent to " $0 \rightarrow\left|\left\{i^{2} \cdot e^{i \pi}\right\} \cdot\left\{i^{2} \cdot e^{i \pi}\right\} \cdot\left\{i^{2} \cdot e^{i \pi}\right\} \cdot\left\{i^{2} \cdot e^{i \pi}\right\} \cdots\right| \rightarrow\left|\left\{i^{2} \cdot e^{i \pi}\right\} \cdot\left\{i^{2} \cdot e^{i \pi}\right\} \cdot\left\{i^{2} \cdot e^{i \pi}\right\} \cdot\left\{i^{2} \cdot e^{i \pi}\right\} \cdots\right| \times i^{2}=-1$ or $e^{i \pi}=-1$ $\rightarrow 0 . "$

\section{Metaphysical Figure, One thing And Noumenon in a Positive SenSE}

Furthermore, we have to say that since the "relation-numbers," i.e., $\left\{i^{2} \cdot e^{i \pi}\right\}$ can serve as "true correlate, i.e., the thing in itself" for space-time-determination in the domain, a metaphysical figure itself disappears in nullity in space-time, concurrently with the event that the "relation-numbers" - 
$\left\{i^{2} \cdot e^{i \pi}\right\}$ - dissolve into "number $\left(\mathbb{N}=\{1,2,3,4, \ldots\}\right.$ and $\left.i^{2}=-1\right)$." We think that 1$)$ number " 1 " or prime number or one and itself in the domain disappears into " 0 " in such a way that " $1 \rightarrow\left\{i^{2} \cdot e^{i \pi}\right\} \times i^{2}$ $=-1$ or $\left\{i^{2} \cdot e^{i \pi}\right\} \times e^{i \pi}=-1 \rightarrow\{1-1\}=0$," which indicates that "real opposition always obtains where $A$ $-B=0$, i.e., where one reality, if combined in one subject with another, cancels out the effect of the latter" (B329), 2) the converse domain signifies the extended field of the domain, i.e., the field of the converse moment of gravity, 3) number "1" or prime number or one and itself in the domain signifies "the series" (B445) - "the manifold of sensible representation (intuition)" which is "represented and apprehended by us as extensive" and the manifold of given representations which is "already intuited as aggregates (multitudes of antecedently given parts)." Number "1" or prime number or one and itself in the domain signifies a metaphysical figure itself - "empirical intuition" (A22), i.e., pure intuition to which sensation is attached - while that in the converse domain signifies the image of a metaphysical figure itself - "a pure image of sensibility" (B377) or "beings of understanding (noumena)." "A pure image of sensibility" is "to be encountered in the mind a priori, wherein all of the manifold of appearances is intuited in certain relations" (A20). Therefore, when a certain relation "occurs a priori, even without an actual object of the senses or sensation" (A21), the relation "Planck's constant" - must pertain to a priori intuition, to which the sensation of empty spacenullified time - sensation in itself - is attached. Since the utter dismemberment or "decomposition" (A525/B553) of "the manifold that is given in a sensible intuition" (B143) is homogeneous with the dissolution of "beings of sense (phaenomena)" (B306), "beings of sense" could dissolve into nullity in space-time - space-time itself - in concurrence with the cessation of "the synthesis of the manifold" (B154). Here we have to acknowledge that the dissolution of beings of sense - the dissolution of $\left\{i^{2} \cdot e^{i \pi}\right\}$ - necessarily corresponds to the cessation of the synthesis of the manifold. Since "a priori intuition" belongs to "a pure category, in which abstraction is made from any condition of sensible intuition," which is necessarily accompanied by "sensation in itself," we think that 1) pure intuition to which sensation in itself is attached, is, in a manner of reciprocity-converse, equivalent to a priori intuition to which sensation in itself needs to be attached, 2) since number " 1 " or prime number or one and itself in the domain necessarily and universally goes through " 0 ," "beings of sense (phaenomena)" - pure intuition to which sensation in itself is attached - are to pertain to "a true object" (A252), i.e., space-time itself, 3) the space-time itself could be correspondent to "number ( $\mathbb{N}=$ $\{1,2,3,4, \ldots\}$ and $i^{2}=-1$ ) or the converse moment of gravity or the (converse) light itself. From this it follows that we say that 1) "a priori intuition," which signifies "a pure image of sensibility" - "beings of understanding (noumena)" - is the "synthetic a priori proposition" (B228) "wherein all of the manifold of appearances is intuited in certain relations" (A20), 2) a priori intuition, i.e., "a pure category" (B304) or the pure intuitions, i.e., "a priori categories" (B159) pertain to "a true object" (A252), i.e., "a thing in itself" (A525/B553) which could consist in "number $(\mathbb{N}=\{1,2,3,4, \ldots\})$ and $i^{2}=-1$." We would like to put it another way, saying that "an unknown something" (B312) or "a something $=X$ " (A250) - "a thing in itself" - can be linked with "beings of sense (phaenomena)" (B306) through "a third thing," namely the "entirely undetermined concept of a being of understanding" (B307) - "supersymmetry" (PENROSE 2007: 869-933). We would say, in an opposite manner to what Kant says (B312), that "now in this way, sensibility acquires a negative expansion, i.e., it is not limited by 'the mere understanding' (A498)."

In contrast to "the mere understanding," which limits "a manifold of sensibility" (B102), such as "mere appearances" (A49) or the "mere categories" (B288) or "mere representations" (A537/B565), inside "the bounds of experience"(A462/B490) or inside the domain, "the pure understanding (realitas noumenon)" (B320), as the "faculty for bringing forth representations itself" (A51), extends them to inside "the bounds of possible experience" (A644/B672) or to the converse domain by calling "mere appearances" "beings of understanding (noumena)" or by calling the "mere categories" imaginary number or by calling "mere representations" "an object of a non-sensible intuition" (B307), i.e., the possibility itself of possible experience. In other words, Max Planck (1858-1947) - "the receptivity of impressions" (A50/B74) and "the understanding itself" (B127) - could extend elements of itself (an appearance or the category or a representation) to inside "the bounds of possible experience" or to the converse domain under the aegis of "Planck's constant." This indicates that "the moment of gravity" or $i^{2}=-1$ or the light itself is commensurate with the reciprocal-converse moment of gravity or $e^{i \pi}=-1$ or the (converse) light itself, respectively. We would like to say that "in this way synthetic a priori judgments are possible" (A158) in regard to the infinite divisibility and decomposition to infinity or 
"the mathematical categories" (B110) or "matter" since a third thing, e.g., " $\left\{i^{2} \cdot e^{i \pi}\right\}$," in virtue of the pure understanding (realitas noumenon) and beings of understanding (noumena)," is to relate the "conditions of a priori intuition, the synthesis of the imagination, and its necessary unity in a transcendental apperception to a possible cognition of experience in general" (A158). In view of the fact that "number" - the pure schema of magnitude - signifies "a correlate of things that subsists by itself" (A487/B515), it is possible to think that the dynamical categories - quantum discretum - are homogeneous with "the appearance as quantum continuum" (A527/B555). That "the conditions of the possibility of experience in general are at the same time conditions of the possibility of the objects of experience" (A158), i.e., that "the field of possible experience" corresponds to "objects of possible experience, has been proven in accordance with rules of a mathematical synthesis. The conditions necessarily stand under "a one-one relation whose domain is the one class and whose converse domain is the other. In such a case we say that there is a 'one-one correlation' between the two classes" (RUSSELL 1971: 52-62). Here, we think that 1) "the one class" is meant to be "objects in themselves" in the domain, i.e., in "the physical world" - "real number $\left(i^{2}=-1\right.$ and $\mathbb{R}=\{1,2,3$, $4, \ldots\})$ " - while "the other" is to be "objects in themselves" in the converse domain, i.e., in the Platonic mathematical world - natural number $(\mathbb{N}=\{1,2,3,4, \ldots\})$ and $e^{i \pi}=-1$ (i.e., the converse $\left.i^{2}=-1\right)-2$ ) there is a "one-one correlation between the two classes" - "the cognition itself a priori in intuition," namely the mental world. From above, it follows that we can say that "objects in themselves" manifest in the mental world as "the field or the entire object of possible experience" (A95), which corresponds to "the entire field of experience" (A533/B561), namely the physical world or as the possibility itself of possible experience or the possibility of experience itself," i.e., the Platonic mathematical world. Once the "transcendental distinction" (A45) between "beings of sense (phaenomena)" and "beings of understanding (noumena)" in the mental world is dissipated, a human can cognize "true correlate, i.e., the thing in itself" and "a thing in itself which one thought through pure concepts of the understanding" (A525/B553), such as death itself or the moment of gravity or the light itself, offering the "ground for the possibility of a priori mathematical cognitions" (B57) Planck's constant and wavefunctions. In view of the fact that the transcendental "ground for the possibility of a priori mathematical cognitions" is parallel to the "synthesis of apprehension" (A102) and "the synthesis of reproduction" (A102), which must take place in one and itself, namely must "take place simultaneously in one and the same occurrence" (A336/B564), we can think that number "1," namely $\left|\left\{i^{2} \cdot e^{i \pi}\right\} \cdot\left\{i^{2} \cdot e^{i \pi}\right\} \cdot\left\{i^{2} \cdot e^{i \pi}\right\} \cdot\left\{i^{2} \cdot e^{i \pi}\right\} \cdots\right|$ arises from the metaphysical path, i.e., synthesis speciosa and transcendental path, i.e., synthesis intellectualis. Therefore, when Kant refers to "empiricism," saying, "empiricism offers advantages to the speculative interests of reason, which are very attractive and far surpass any that the dogmatic teacher of the ideas of reason might promise. For with empiricism the understanding is at every time on its own proper ground, namely the field solely of possible experiences, whose laws it traces, and by means of which it can endlessly extend its secure and comprehensible cognition. Here it can and should exhibit its object, in itself as well as in its relations, to intuition, or at least in concepts an image for which can be clearly and distinctly laid before it in similar given intuitions. Not only is it unnecessary for the understanding to abandon this chain of natural order so as to hang onto ideas with whose objects it has no acquaintance" (A468/B496-A469/B497), we agree with him, thinking that empiricism offers advantages to "the architectonic interest of reason" (A475/B503) and "the speculative interests of reason," namely "a merely dialectical conflict" (A505/B533). Since ideas with whose objects the understanding appears to have no acquaintance, such as "the cosmological idea" (A489/B517) or "two eternal and infinite self-subsisting non-entities (space and time)" (B56) could "be generated in accordance with rules of a mathematical synthesis" or "the mathematical principles" (A149), "mathematical-transcendental ideas" (A529/B557) are "not even permitted to abandon its business, and, under the pretext that this has been brought to an end, to pass over into the territory of" (A469/B497) transcendental dialectic, "where there is no further need to make observations and to inquire according to the laws of nature, but rather only to think and invent" (A469/B497). Therefore, we have to say, in an opposite manner to what Kant refers to (A644/B672), saying, "Accordingly, we assert: the transcendental ideas such as the infinite divisibility or decomposition to infinity are of constitutive use, so that the concepts of certain objects would thereby be given and in case one so understands them, they are the "possible empirical concept' (A489/B517) - a synthetic a priori concept. In addition, they have an excellent and indispensably necessary regulative use, namely that of directing the understanding to a certain goal respecting which the lines of direction of all its rules converge at one point, which is not the point 
called focus imaginarius, but 'the center of the circle' (EUCLID 2002: 1-36), which lies entirely inside the bounds of possible experience, from which the concepts of the understanding proceed."

In Kant's metaphysics, "things in themselves" are endowed with double meanings: either "things in themselves" which are "entirely beyond our cognitive sphere" (A190) or the "true correlate, i.e., the thing in itself." Therefore, Kant tries to divide "a noumenon" into two: "a noumenon in the negative sense" (B307) and "the noumenon in a positive sense" (B307). Since the former is thought to pertain to "a thing insofar as it is not an object of our sensible intuition, because we abstract from the manner of our intuition of it" (B307), while the latter is thought to pertain to "an object of a non-sensible intuition" (B307), i.e., "a special kind of intuition, namely intellectual intuition, which, however, is not our own" (B307), we can say that 1) the noumenon in a positive sense, i.e., an object of a nonsensible intuition, such as the light itself or the moment of gravity, is homogeneous with "the pure concept," which has its origin in the understanding and in a pure image of sensibility, 2) it is to progress - "progressus in indefinitum" (A511/B539) - to a something $=X$, i.e., the "transcendental object" or "a true object," such as the (converse) light itself or the converse moment of gravity, indicating that the "progressus in indefinitum" is commensurate with going "back to infinity" (A514/B542) - the "regressus in infinitum" (A514/B542). Thus the noumenon in a positive sense, which is to progress, namely to regress, to the transcendental object, is to be parallel to "a noumenon in the negative sense." Thus we can think that "the noumenon in a positive sense" and "a noumenon in the negative sense" - the "transcendental schema" (A138/B177) and the transcendental object belong to "synthetic a priori propositions" - "axioms." Therefore, when Kant says, "That which we call noumenon must be understood to be such only in a negative sense" (B309), we entirely agree with him. Seeing that "beings of understanding (noumena)," i.e., a something $=X-$ "the pure category" (A245) - can "contain nothing but the logical function for bringing the manifold under a concept" (A245), "the logical function," e.g., Leonhard Euler could enhance "some sort of concept of intelligible things" (A566/B594) (e.g., inductive numbers) - the analogy of "all appearances" (B204), which "are accordingly already intuited as aggregates (multitudes of antecedently given parts) (B204) - to the mathematical-transcendental ideas, namely "dynamical" categories (thereby mathematical categories) by means of the "zeta-function" and " $e^{i \pi}+1=0$." Thus, contrary to Kant's way of thinking that the pure concept or the pure category has "its origin solely" in pure a priori concepts the noumenon in a positive sense - we have to think that the pure concept has its origin in "a noumenon in the negative sense" - the pure category (e.g., the converse moment of gravity). Here, it is critically important to acknowledge that though the pure concept or the pure category - a thing in itself - cannot go beyond "the bounds of possible experience," it "acquires a negative expansion" (B312). In view of the fact that the noumenon in a positive sense - "a thing in itself" - is inhaled into "a noumenon in the negative sense" - "the field of possible empirical cognition" (A644/B672) - we can think that "the concept of a noumenon" (A256) signifies "a concept setting limits to sensibility" (A256), e.g., the "Dedekind gap" (RUSSELL 1971: 63-76) or "a boundary concept" (A255-B311), e.g., a "Dedekind cut" (RUSSELL 1971: 63-76), which is to "extend sensible intuition to things in themselves" (B310), and thus not "to limit the objective validity of sensible cognition" (B310).

Thus, a concept setting limits to sensibility - the Dedekind gap - seems parallel to " $\aleph_{0}$ terms, i.e., $e^{i \pi}$ $=-1=i^{2 "}$ " "a priori intuition" or Planck's constant - while a boundary concept - a Dedekind cut, i.e., $\left\{i^{2} \cdot e^{i \pi}\right\}$ - seems parallel to "intuitions themselves" and "perceptions themselves" - "the transcendental unity of the synthesis of the imagination" (A118) - wavefunctions. Since a Dedekind cut, i.e., $\left\{i^{2} \cdot e^{i \pi}\right\}$ signifies the moment of gravity and the converse moment of gravity, we can say that a Dedekind cut, i.e., $\left\{i^{2} \cdot e^{i \pi}\right\}$ - wavefunctions - or the Dedekind gap, i.e., " $e^{i \pi}=-1=i^{2 "}$ " Planck's constant - is generated through the "attempted physiological derivation" (B119-A87), namely the infinite divisibility and decomposition to infinity, which is meant that "their intuition and the real in their perception could be generated" (B221) in accordance with "the objects of nature itself." It is not "a quaestio facti, the explanation of the possession of a pure cognition" (A87) but the explanation of the possession of a synthetic a priori cognition. What does it mean? It means that "intuitions themselves" - a priori intuition and pure intuition - are possessed of "perceptions themselves" - a priori categories (e.g., the intensive magnitude) and the pure category (e.g., the extensive magnitude). When we cognize this, we have to think that "pure schema of magnitude (quantitatis)," i.e., "number" is nothing but the concepts of the understanding, which rests on synthetic a priori cognition. When Kant refers to pure a priori concepts, saying, "Yet a deduction of the pure a priori concepts can never be 
achieved in this way" (B119), we say that, in view of the fact that pure a priori concepts - "number" are homogeneous with pure a priori cognitions (i.e., pure a priori intuition) which is to be parallel to "a synthetic a priori concept," "a deduction of the pure a priori concepts" can be achieved in conjunction with a deduction of the synthetic a priori concept, under which "rules of a mathematical synthesis" of things in themselves stand. Therefore, we say that the transcendental deduction of "objects of possible experience" (B166) in conjunction with the "metaphysical deduction" (B159) of "the origin of the a priori categories" (B159) corresponds to the "attempted physiological derivation."

Furthermore, we would like to say that 1$)$ the elements of the domain $\left(\left\{i^{2} \cdot e^{i \pi}\right\}=1\right)$ necessarily go through a "metaphysical path" to the converse domain $\left(e^{i \pi}=-1=i^{2}\right)$ while the elements of the converse domain $\left(e^{i \pi}=-1=i^{2}\right)$ necessarily go through "transcendental path" to the domain, 2$)$ the "metaphysical path" and the "transcendental path" have a one-one relation in a manner of instantaneous and simultaneous reciprocity-converse, 3) "relation-numbers" $-\left\{i^{2} \cdot e^{i \pi}\right\}=1$ " - which are homogeneous with the "metaphysical path" and "transcendental path," signify "a thoroughgoing connection in one experience" (A146), namely "the systematic in cognition, i.e., its interconnection based on one principle" (A645/B673). Since " $e^{i \pi}=-1=i^{2}$ and $\left\{i^{2} \cdot e^{i \pi}\right\}=1$ " can be thought to signify "two eternal and infinite self-subsisting non-entities (space and time)" (B56), i.e., "relations of appearances (next to or successive to one another) that are abstracted from experience" (A40-B57), the origin of them "must really be sought in experience, out of whose abstracted relations imagination has made something that, to be sure, contains what is general in them but that cannot occur without the restrictions that nature has attached to them" (B57). A metaphysical figure itself - "a transcendental concept of reason" (A326) - never ends "with the absolutely unconditioned, i.e., what is unconditioned in every relation" (A326) - the Dedekind gap, i.e., " $e^{i \pi}=-1=i^{2}$ " - which could be homogeneous with natural number $(\mathbb{N}=\{1,2,3,4, \ldots\})$ and $i^{2}=-1$ but "goes to the absolute totality in the synthesis of conditions" (A326) - a "Dedekind cut," i.e., $\left\{i^{2} \cdot e^{i \pi}\right\}-$ by means of "pure mathematics." In regard to the possibility of pure mathematics, we have already said above that "an $a$ priori judgment in regard to the infinite divisibility and the given judgment in regard to decomposition to infinity are the 'ground for the possibility of a priori mathematical cognitions' (B57) - the ground for 'pure mathematics' (A39) and 'a priori mathematical doctrines in regard to real things' (B57)." This is "the principles absolutely" (B358) - "the supreme principle of all synthetic judgments" (A158). In view of the fact that the "transcendental path" and the "metaphysical path" pertain to the "origin of the a priori categories" (B159) and "a priori cognitions of objects of an intuition" (B159), we dare to say that the "paths" correspond to $\zeta(\mathrm{s})=0$ ("complex zeros of the zetafunction" (CONREY 2003: 341-353) in the Riemann zeta function. Since $\zeta(\mathrm{s})=0$ haunts when 1) there is no $i$ or $i^{2}$, and 2) $x$ in $\zeta(\mathrm{s})$ is " 1 ," indicating that $\zeta(\mathrm{s})=0$ could be the "paths" where the "synthesis of apprehension" (A102) and "the synthesis of reproduction" (A102) take place "simultaneously in one and the same occurrence" (A336/B564) in "everything that exists simultaneously in the various positions in space, no matter how similar and equal they might otherwise be" (B320), the "paths" yield the category or the categories, which signify nothing but "one thing (numerica identitas)" (B319).

Therefore, we make "synthetic propositions" (B18), which state that 1) there are two modes of " 0, " 2) one " 0 " corresponds to "a pure category, in which abstraction is made from any condition of sensible intuition" (B304), which consists in the Riemann zeta function $\zeta(\mathrm{s})$, in which $x$ is " $0<x<1$ " and there is a free-floating $i$ or $\left.i^{2}, 3\right)$ the function $\zeta(\mathrm{s})$, in which $x$ is " $0<x<1$ " and there is a free-floating $i$ or $i^{2}$, signifies the "converse" prime number or the (converse) light itself or the converse moment of gravity, 4) the other " 0 " corresponds to the "paths," in which the "pure synthesis of the imagination" (A118) takes place, yielding, "simultaneously in one and the same occurrence," the moment of gravity and the converse moment of gravity, 5) the "paths" signifies the "rules of synthetic a priori unity" (A217) - the "transcendental unity of the synthesis of the imagination" (A118), i.e., " $\left\{i^{2} \cdot e^{i \pi}\right\}=1$," which is "necessary a priori" (A118). This is our "synthetic propositions" in regard to the "paths" supersymmetry - which pertain to "abstracted relations" and "mere relations" (B321). We have to take note of the facts that 1) the former " 0 " - the converse domain - comprises all elements of "relation-numbers" $-\mathbb{N}=\{1,2,3,4, \ldots\}$ and $i^{2}=-1-2$ ) the domain, which consists in "objects of experience" (B258) and "objects of sensibility," corresponds to "the sum total of all appearances (natura materialiter spectata)" (B163), 3) therefore, it comprises all elements of "relation-numbers" $\mathbb{N}=\{1,2,3,4, \ldots\}$ and $i^{2}=-1$ - which correspond to real number $\left(i^{2}=-1\right.$ and $\left.\mathbb{R}=\{1,2,3,4, \ldots\}\right)$. From 
the discourse so far, it follows that it is possible to say that 1) "the absolutely unconditioned, i.e., what is unconditioned in every relation" - the Dedekind gap, i.e., " $e^{i \pi}=-1=i^{22}$ - is correspondent to empty space-nullified time, i.e., space-time itself, 2) since a filled space-elapsing time concerns alteration, it pertains to "the combination of contradictorily opposed determinations in the existence of one and the same thing" (B291) and "a combination of contradictorily opposed predicates...in one and the same object" (B48), 3) since a "series of appearances (alterations)" (B281) signify "the unconditioned, which is conditioned by $\left\{i^{2} \cdot e^{i \pi}\right\}$ " - "what is possible in all respects in every relation" (B381), namely "potentialiter infinite" (B446) - it could be commensurate with "oneness itself," i.e., " $\left\{i^{2} \cdot e^{i \pi}\right\}=1$ "

Furthermore, we say that 1) the converse domain - "the absolutely unconditioned, i.e., what is unconditioned in every relation" - could be commensurate with " $e^{i \pi}=-1=i^{2}$," namely " $e^{i \pi}+1=0=i^{2}$ $+1, " 2)$ the "entire universe," i.e., "the absolute whole of magnitude (the world-whole)," which consists in the converse domain and the domain, corresponds to appearances themselves, i.e., filled space-elapsing time $\left(\left\{i^{2} \cdot e^{i \pi}\right\}=1\right)$ and empty space-nullified time $\left(e^{i \pi}=-1=i^{2}\right.$, i.e., " $\left.e^{i \pi}+1=0=i^{2}+1 "\right)$. However, the entire universe lacks "the schema of community (reciprocity), or of the reciprocal causality of substances" (A144) - the moment of gravity and the converse moment of gravity - with regard to itself. In other words, there is no "juxtaposition of the manifold of empirical intuition" (B219) with regard to it. Therefore, the entire universe, which does not stand under the "reciprocal causality of substances," remains "the possibility of the category of community" (B292), indicating that it does not obey "the second law of thermodynamics" (BENNETT 1987: 108-116). In contrast to the entire universe, filled space-elapsing time $-\left\{i^{2} \cdot e^{i \pi}\right\}=1-$ signifies "the unconditioned, which is conditioned by the paths" - "realitas phaenomenon" (A265) and "a substantia phaenomenon" (B321). However, it does not obey the second law of thermodynamics as well because it comprises the paths $\left\{i^{2} \cdot e^{i \pi}\right\}$ - which seems to be parallel to Maxwell's demon (BENNETT 1987: 108-116). We have to add, saying that since the entire universe, while constituting "the field or the entire object of possible experience," lacks "a priori principles of the possibility of experience," e.g., "allness (totality)" (B111) or "unity of experience" (A180), "we must progress further to this condition; this is a regressus in indefinitum" (A520/B548). In view of the facts that 1) a filled space-elapsing time - one and itself - stands under synthesis speciosa which pertains to "itself" and synthesis intellectualis which pertains to "one," 2) a filled space-elapsing time - one and itself - signifies a thing "that exists simultaneously in the various positions in space" (B320) - the "possibility itself" of one thing - while the entire universe $\left(e^{i \pi}+1=0=i^{2}+1\right.$ and $\left.\left\{i^{2} \cdot e^{i \pi}\right\}=1\right)$ cannot signify a thing, we have to think that a world-whole itself - the entire universe - signifies "the possibility of a world-whole itself" (A677/B705) - mere "possibility of the category of community" - while "one and itself" signifies "possibility itself" of the category of community. If we go to the entire universe by means of "number" and "numerical formulas," it remains "the possibility of the category of community" or "the possibility of things in the world of sense" (A677/B705) though the regress in it (e.g., the zeta function) appears to be complete. If we go to a filled space-elapsing time by means of "number" and "numerical formulas," the regress in it $\left(\left\{i^{2} \cdot e^{i \pi}\right\}\right)$ is complete (namely number " 1 "), thereby its being the "possibility itself" (B111) of the category of community or the possibility itself of things in the world of sense. Thus "possibility itself" pertains to the "transcendental ideality of appearances" - "a projected unity" (A647/B675) - while "possibility" appears to pertain to "an ens extramundanum" (A561/B589). However, since "possibility" and "possibility itself" arise through "the mathematical regress" which "really has to do only with the combination of parts into a whole, or with the dissolution of a whole into its parts" (A560/B588) and "the dynamic regress" (A560/B588), which has to do with "the possibility of an unconditioned whole or an unconditioned part of a given whole" (A560/B588), "the conditions of this series always have to be seen as parts of it, hence as being of the same kind" (A560/B588), indicating that "the infinite divisibility" is commensurate with "decomposition to infinity." Therefore, we have to think that 1) "the condition need not necessarily constitute one empirical series along with the conditioned" (A560/B588), 2) the condition $\left(e^{i \pi}+1=0=\right.$ $i^{2}+1$ and $\left\{i^{2} \cdot e^{i \pi}\right\}=1$, namely Planck's constant and wavefunctions) is either "the derivation of a state from its cause or of the contingent existence of a substance itself from the necessary existence of one" (A560/B588). Thus our "cosmological ideas" (B438), which are concerned with "the regress of the decomposing synthesis," go "in antecedienta" (B438) and "in consequentia" (B438). We think that first-order logic - "the regressive synthesis itself" and "the regress itself" - which comprise an "instantaneous and simultaneous reciprocity-converse," i.e., supersymmetry is the "only one way out of the apparent antinomy lying before us" (A560/B588). 


\section{REFERENCES}

Bennett, Charles H, “Demons, Engines and the Second Law," Scientific American, November 1987, pp. 108116, 1987.

Conrey, J Brian, “The Riemann Hypothesis,” Notice of the AMS 50: 341-353, 2003.

Euclid, Euclid's Elements, Green Lion Press, 2002.

Frege, Gottlob, The Foundations of Arithmetic, Northwestern University Press, 1980.

Gödel, Kurt, "On Formally Undecidable Propositions of Principia Mathematica and Related Systems I," The Undecidable, Dover Publications, pp. 5-38, 2004.

Gödel, Kurt, "Remarks Before the Princeton Bicentennial Conference on Problems in Mathematics - 1946 - " The Undecidable, Dover Publications, pp. 84-88, 2004.

Hilbert, David, "Mathematical Problems," Bulletin of the American Mathematical Society 8: 437-479, 1902.

Hodel, Richard E, An Introduction to Mathematical Logic, Dover Publications, 2013.

Kant, Immanuel, Critique of Pure Reason, Cambridge University Press, 1999.

Penrose, Roger, The Road to Reality: a Complete Guide to the Laws of the Universe, Vintage Books, 2007.

Russell, Bertrand, Introduction to Mathematical Philosophy, Simon \& Schuster, 1971.

Snapper, Ernst, "The Three Crises in Mathematics: Logicism, Intuitionism and Formalism," Mathematics Magazine 52: 207-216, 1979.

Tanton, James, Encyclopedia Mathematics, Fact On File, 2005

Wigner, Eugene P, "The Unreasonable Effectiveness of Mathematics in the Natural Sciences," Communications on Pure and Allied Mathematics 13: 1-14, 1960.

Yamamoto, Kazuhiko, "The Pure Concepts of the Understanding and Synthetic A Priori Cognition: the Problem of Metaphysics in the Critique of Pure Reason and a Solution," Proceedings of the European Conference on Ethics, Religion \& Philosophy 2016, pp 87-100, 2016.

Yamamoto, Kazuhiko, "The Transcendental Aesthetic and Absolute Totality of Conditions: The Problem of Metaphysics in the Critique of Pure Reason and a Solution," International Journal of Humanities Social Sciences and Education 4 (2): 19-37, 2017a.

Yamamoto, Kazuhiko, "Absolute Totality, Causality, and Quantum: The Problem of Metaphysics in the Critique of Pure Reason," International Journal of Humanities Social Sciences and Education 4 (4): 72-81, 2017b.

Yamamoto, Kazuhiko, "Numbers, Geometry, and Mathematical Axioms: The Problem of Metaphysics in the Critique of Pure Reason," International Journal of Humanities Social Sciences and Education 4 (6): 57-70, $2017 \mathrm{c}$.

Yamamoto, Kazuhiko, "Categories, Causality, and Numbers: The Problem of Metaphysics in the Critique of Pure Reason," International Journal of Humanities Social Sciences and Education 4 (8): 19-29, $2017 \mathrm{~d}$.

Yamamoto, Kazuhiko, "The Real, the Infinite, Categories, and Cardinal Numbers: The Problem of Metaphysics in the Critique of Pure Reason," International Journal of Humanities Social Sciences and Education 5 (4): 20-45, 2018.

Citation: Kazuhiko Yamamoto. "Transcendental Logic, One and Itself, First-Order Logic and Number: The Problem of Metaphysics in the Critique of Pure Reason". International Journal of Humanities Social Sciences and Education (IJHSSE), vol. 6, no 8, 2019, pp. 43-70. doi: http://dx.doi.org/10.20431/2349-0381.0608005.

Copyright: (1) 2019 Authors. This is an open-access article distributed under the terms of the Creative Commons Attribution License, which permits unrestricted use, distribution, and reproduction in any medium, provided the original author and source are credited. 